\title{
Study of the Properties and Interactions of Elementary Particles
}

\author{
Summary \\ Grant DE-FG02-95ER40899 \\ University of Michigan \\ Department of Physics \\ Ann Arbor, Michigan 48109-1040
}

Principle Investigator: Dante Amidei 734/764-3266 amidei@umich.edu

DOE/Office of Science Program Office : High Energy Physics

DOE Technical Program Manager Contact: Dr. Alan Stone

Submitted August, 2012 


\section{Final Report \\ Study of the Properties and Interactions of Elementary Particles \\ The University of Michigan \\ Summary of period 2009-2011 \\ DE-FG02-95ER40899 \\ PI: Dante Amidei \\ amidei@umich.edu}

We summarize the accomplishments of the last renewal period in a broad program of High

Energy Physics research at the University of Michigan, as supported by the U.S. Department of

Energy.

\section{Energy Frontier}

Page

Task A: The ATLAS group reports on the search for the light Higgs boson (and finding it!), as well as the study of multiple vector boson production, lambda polarization and SUSY searches in the 7-8 TeV p-p collisions at the LHC.

Task C: The CDF group reports results on two Tevtron anomalies: a large, mass-dependent forward-backward asymmetry in $t \bar{t}$ production and a $b \bar{b}$ resonance, consistent with a SUSY Higgs particle, produced in association with another $b$.

Task K: The D0 group completed the search for the Higgs boson in the $\mathrm{WH}$ and $\mathrm{ZH}$ final states, the measurement of the weak-mixing angle $\sin ^{2} \theta_{W}^{l}$ using the $Z / \gamma^{*}$ asymmetry, and a search for gauge mediated SUSY in $Z \gamma+$ missing energy events.

\section{Intensity Frontier}

Task C: The K0TO group describes progress toward measurement of $K_{L} \rightarrow \pi^{0} \nu \bar{v}$ at experiment E14 at J-PARC, including critical contributions to the design and construction of the trigger, data acquisition, and veto systems for the CsI crystal array.

\section{Cosmic Frontier}

Task I: The Dark Energy group reports on preparations for the systematic study of dark energy at the DES telescope, progress on NIR detector development, and preparations for the next generation of dark energy measurements at BigBoss.

\section{Theory}

Task G: The High Energy Theory group reports on a broad program of investigations in the areas of particle phenomenology in the LHC era, particle cosmology in the era of direct and indirect detection of dark matter, and both foundational and phenomenological approaches to string theory.

Task J: The Cosmology and Fundamental Physics group reports predictions of dark energy models, as well as studies of optimizing the observations provided by DES, BigBOSS and other cosmological surveys in order to understand the nature of dark energy. 


\title{
DOE Task A (ATLAS) Closeout Report (2009 - 2012)
}

\author{
J. Chapman, H. Neal, J. Qian, R. Thun, B. Zhou, J. Zhu \\ T. Dai, E. Diehl, D. Levin, X. Li, S. McKee, N. Panikashvili, H. Yang, A. Wilson, G. Wood \\ A. Armbruster, D. Harper, H. Liu, L. Liu, J. Long, J. Purdham, D. Scheirich, S. Walch \\ August 15, 2012
}

\section{Executive Summary}

The last three years have been extremely exciting for the field of experimental high energy physics. The Large Hadron Collider (LHC) started pilot operation of proton-proton collisions in Nov. 22, 2009. During 2010 and 2011 the LHC operated with beam collisions at a center-of-mass energy of $7 \mathrm{TeV}$. The centerof-mass energy has been further increased to $8 \mathrm{TeV}$ in 2012. The total integrated luminosity delivered by the LHC to the ATLAS experiment to date has exceeded $16 \mathrm{fb}^{-1}$, with a peak instantaneous luminosity of $6.8 \times 10^{33} \mathrm{~s}^{-1} \mathrm{~cm}^{-2}$. The operations of the ATLAS detector during the first three years have been remarkable: over $99 \%$ of the detector channels were fully functional and the online data-taking efficiency exceeded 95\%. This Fourth of July, while the United States celebrated Independence Day, scientists at CERN, near Geneva, Switzerland, including members of the University of Michigan ATLAS group, announced one of the most important discoveries in fundamental physics, the detection of a new particle with properties consistent with the Standard Model Higgs boson.

The University of Michigan's ATLAS research has been coordinated by five faculty members: Neal, Qian, Thun, Zhou and Zhu. Emeritus Professor Chapman also remains very active in ATLAS research. This group has been one of the most active and productive groups in the ATLAS Collaboration and has built a strong reputation for innovation in designing and building large particle detectors and for leadership in the discovery of the Higgs-like boson . We operate one of three world-wide ATLAS muon calibration centers under the leadership of our research scientists Diehl and Levin, provide one of the leading computational centers for the entire experiment under the leadership of our research scientist McKee, and have played a leading role by research staff Dai in the commissioning and operation of the largest muon spectrometer ever constructed. These proved to be critical in the discovery of the Higgs-like boson. Professor Qian coordinated the effort of over one hundred physicists from all over the world as the convener of the ATLAS group searching for the Higgs boson in the WW decay channel and is also a major contributor to the statistical analysis of the combination of Higgs searches from different channels. Professors Zhou and Thun together with research scientist Yang have concentrated their analysis on the diboson (WW, ZZ and WZ) cross-section measurements and on the Higgs search in the ZZ decay channel where a signal was observed at a mass of $126 \mathrm{GeV}$, thereby determining one of the key parameters of the Higgs boson. These analysis efforts have contributed significantly to the claim of the discovery on July 4th. A paper summarizing the discovery has been recently published in Physics Letters B. Professor Neal has concentrated on developing techniques for studying particle quantum spin and will be applying these tools to help confirm that the spin and parity of the newly discovered particle is indeed that required of the Higgs in the Standard Model. Professor Zhu is a leading physicist in ATLAS pursuing the study of vector boson scattering and fusion to test the Higgs mechanism and to search for physics beyond the Standard Model in the diboson final states.

Over the past three years, University of Michigan ATLAS physicists provided leadership roles and major effort to 34 ATLAS published papers, 35 public conference physics notes and 102 ATLAS internal notes. The physics topics span a broad range of areas including detector performance studies, rediscovery of the SM electroweak and B processes at the LHC, searches for supersymmetry, searches for new particles with diboson final states and the discovery of the Higgs-like boson. UM physicists also presented about 70 talks in national and international conferences, HEP seminars and workshops. The physics papers are listed in this report. Other notes and talks can be found in the links listed in this report. In addition, the 
UM ATLAS group organized and hosted an international conference on "The First Year of LHC Data" in December 2010 and the US ATLAS physics workshop in August 2012.

The physics goals of the LHC experiments are to find the origin of mass, the origin of dark matter, and to search for new physics beyond the Standard Model. The discovery of a new boson consistent with the SM Higgs is a historic milestone, but only the beginning of the planned two decades long physics program at the LHC. There are still important and profound physics questions related to the mysteries of the electroweak symmetry breaking and the Higgs sector of the SM. Both accelerator scientists and experimentalists at the LHC are now intensively preparing to address deeper questions and to further advance our understanding of the universe. The LHC energy will be increased to 13-14 TV and the instantaneous luminosity will reach $10^{34} \mathrm{~cm}^{-2} \mathrm{~s}^{-1}$ in 2015 . The University of Michigan ATLAS group looks forward to many years of elucidating the still mysterious Higgs mechanism. Our physics analysis will be focused on the property measurements of the newly discovered boson and studies of vector boson fusion and scattering to test the SM Higgs mechanism. We will also continue to search for new particles decaying to diboson final states and search for dark matter signatures with leptons and missing energy in final states at the LHC. To reach these physics goals, we are fully committed to continue operating and calibrating the ATLAS Muon Spectrometer and to lead the design and production of the muon trigger system upgrade work in coming years.

\section{Selected very recent physics results with major contributions from the UM group}

1) Discovery of the new Higgs-like boson with the $Z Z$ (left) and WW (right) final states. Left plot shows the $\mathrm{ZZ} \rightarrow$ 4lepton mass spectrum; right plots shows the WW transverse spectrum. Major UM working force: Qian, Thun, Zhou, Armbruster, Dai, Li, H. Liu, Long, Strandberg, Wilson, Wood, Wu, Yang

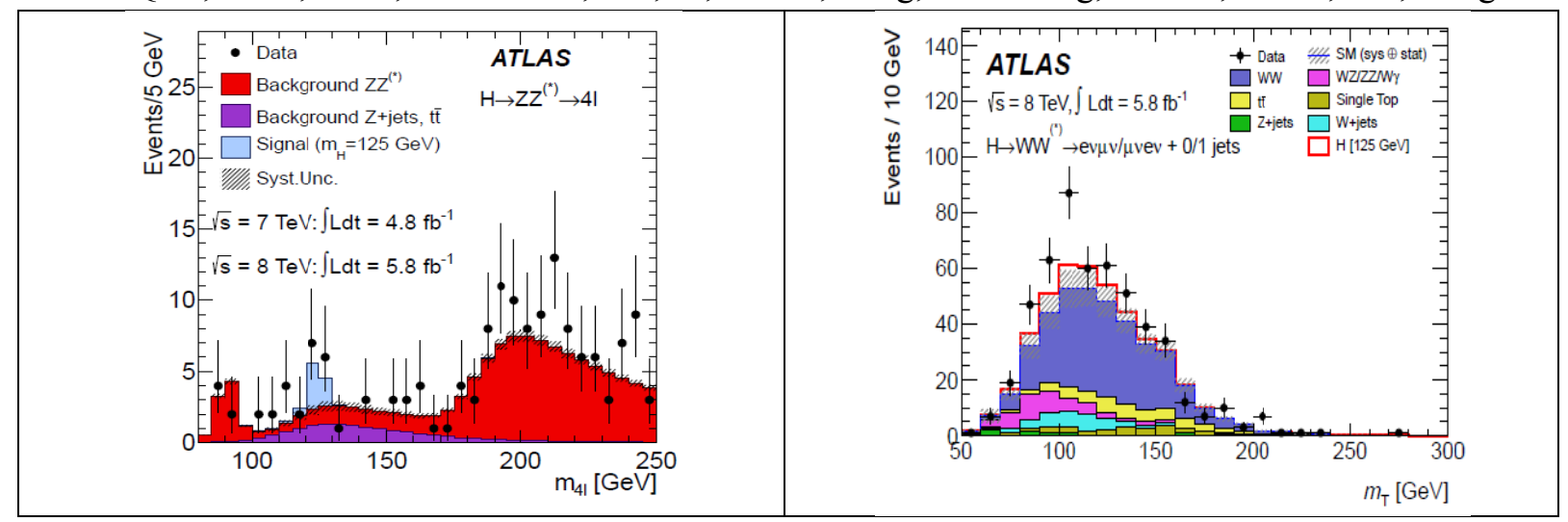

2) Search for new resonances in the WZ (left) and WW (right) final states with leptonic decay channels. Major UM working force: Zhu, Boronni, L. Liu, and $\mathrm{Wu}$

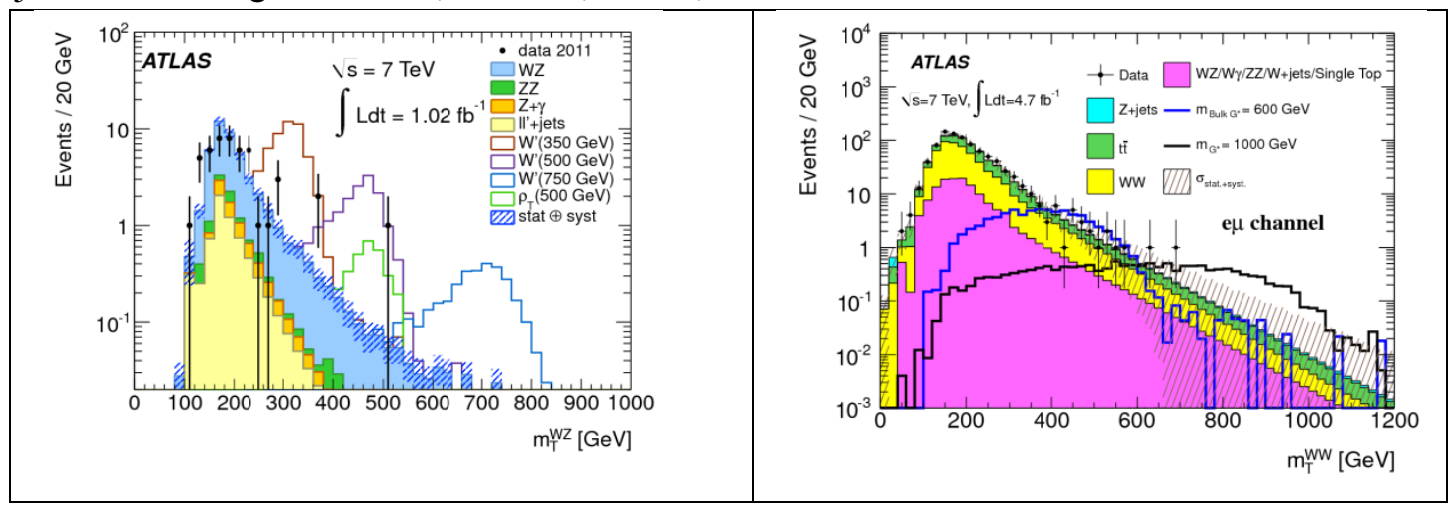


3) Measurement of the production cross-sections. Transverse mass distributions of WW (left), $\mathrm{WZ}$ (middle) and ZZ (right) with leptonic decay final states using full datasets at $7 \mathrm{TeV}$. Major $\mathrm{UM}$ working force: Zhou, Zhu, Thun, Dai, Levin, J. Liu, L. Liu, Wilson, Wu, Xu, Yang
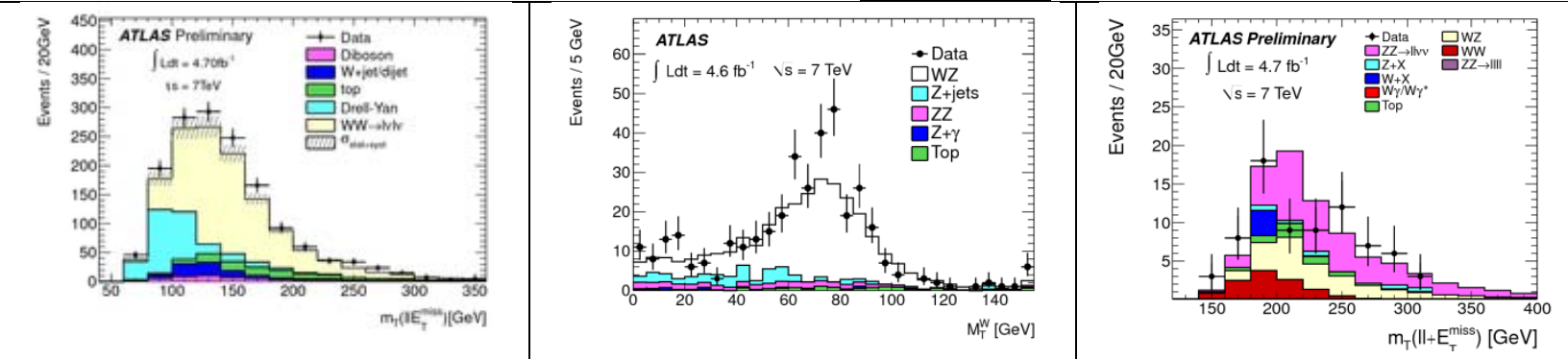

4) The anomalous triple-gauge boson coupling limits from the diboson physics analysis. Major UM working force: Zhou, Zhu, Dai, J. Liu, L. Liu, Wu, Yang and Xu
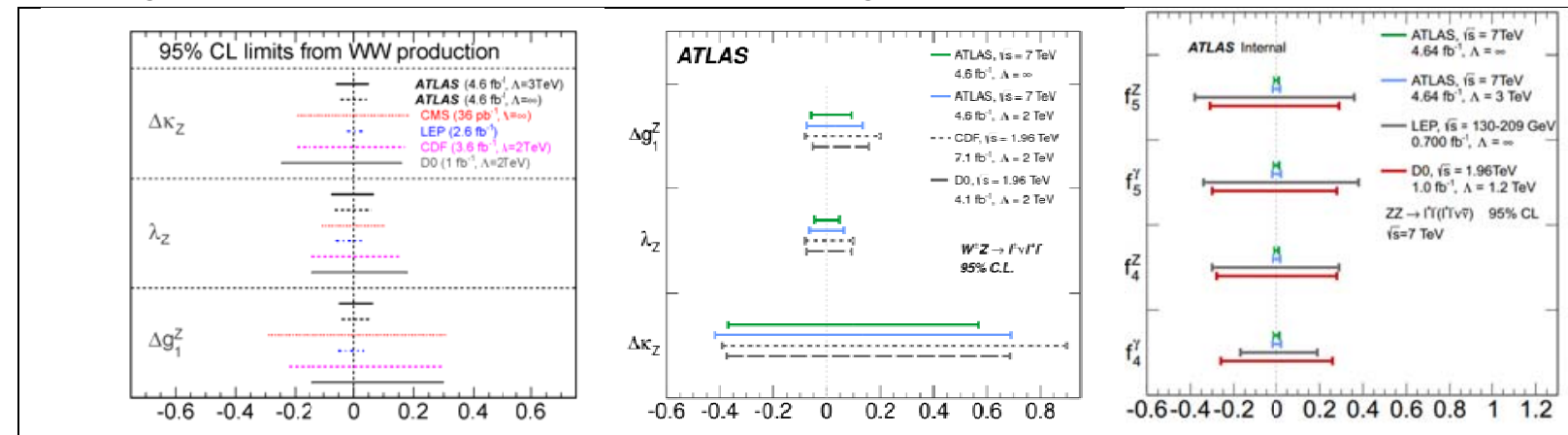

5) $\Lambda_{\mathrm{b}}$ life time measurement (left) and the GMSB SUSY search limit (right) in the higgsino and the gluino mass plane with the experimental signature of $\mathrm{Z}+$ missing $\mathrm{E}_{\mathrm{T}}$. Major UM working force: Neal, Panikashvili, Harper, Scheirich

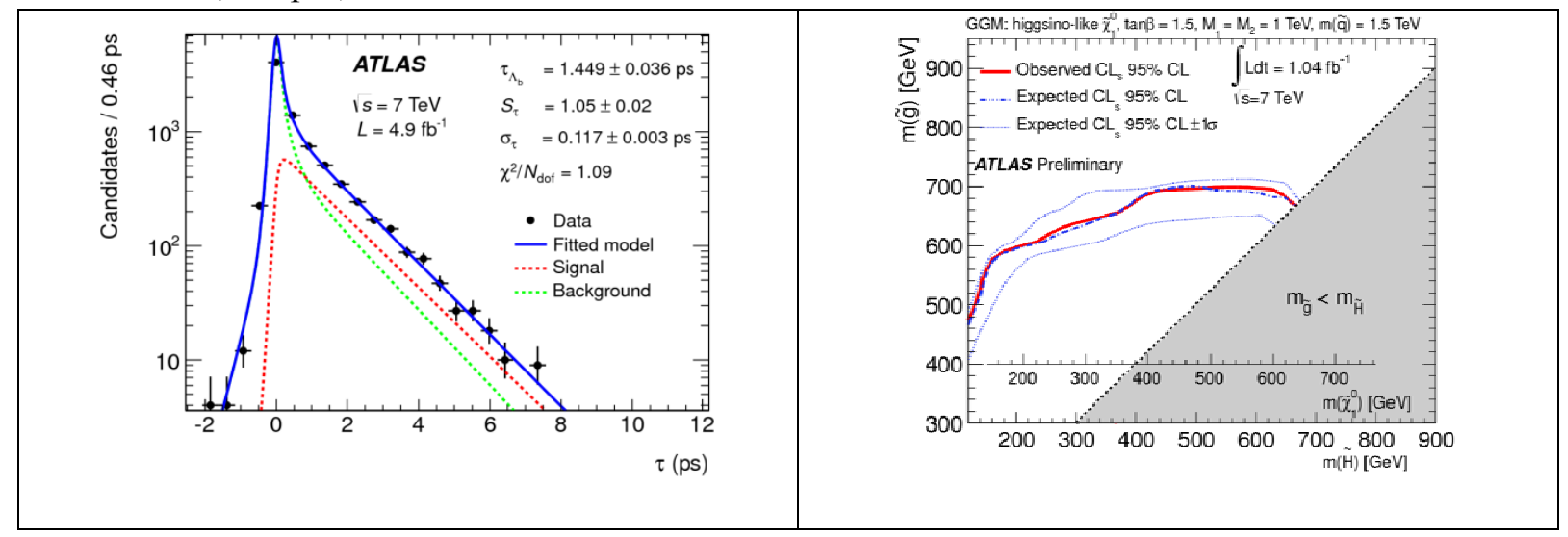

\section{List of ATLAS paper that Michigan group made major contributions (2009-2012)}

1) "Measurement of WZ Production in Proton-Proton Collisions at $\sqrt{s}_{s}=7 \mathrm{TeV}$ with the ATLAS Detector", submitted to EPJC on 8/7/2012 
2) "NEW Observation of a new particle in the search for the Standard Model Higgs boson with the ATLAS detector at the LHC", submitted to PLB on 7/31/2012

3) "NEW Measurement of the Lambda_b lifetime and mass in the ATLAS experiment", submitted to PRD on $7 / 10 / 2012$

4) "Combined search for the Standard Model Higgs boson in pp collisions at $\sqrt{s}_{\mathrm{s}}=7 \mathrm{TeV}$ with the ATLAS detector", Accepted by PRD publication (submitted on 7/2/2012)

5) "Search for new physics in WW $->$ lnulnu final state in pp collisions at sqrt(s) $=7 \mathrm{TeV}$ with the ATLAS detector ", Submitted to Phys Lett B. on 8/14/2012

6) "Search for the Standard Model Higgs boson in the $\mathrm{H}->\mathrm{WW}->$ lvlv decay mode with $4.7 \mathrm{fb}^{-1}$ of ATLAS data at $\sqrt{s}_{\mathrm{s}}=7 \mathrm{TeV}$ ", accepted by PLB (submitted on 6/4/2012)

7) "Search for Lepton Flavour Violation in the emu Continuum with the ATLAS detector in $\sqrt{s}_{\mathrm{s}}=7 \mathrm{TeV}$ pp collisions at the LHC", Eur.Phys.J. C72(2012) 2040

8) "Search for resonant $\mathrm{WZ}$ production in the $\mathrm{WZ}$ to lnul'l' channel in $\sqrt{\mathrm{s}}_{\mathrm{s}}=7 \mathrm{TeV}$ pp collisions with the ATLAS detector",Phys.Rev.D85 (2012) 112012

9) "Measurement of the WW cross section in $\sqrt{\mathrm{s}}_{\mathrm{S}}=7 \mathrm{TeV}$ pp collisions at ATLAS and limits on anomalous gauge couplings", Physics Letters B 712 (2012) 289-308

10) "Combined search for the Standard Model Higgs boson using up to $4.9 \mathrm{fb}-1$ of pp collision data at $\sqrt{\mathrm{s}}_{\mathrm{s}}$ $=7 \mathrm{TeV}$ with the ATLAS detector at the LHC", Phys.Lett. B710 (2012) 49-66

11) "Search for the Standard Model Higgs boson in the decay channel H->ZZ(*)->41 with 4.8 fb-1 of pp collisions at $\sqrt{s}_{\mathrm{s}}=7 \mathrm{TeV}$ with ATLAS", Phys.Lett. B710 (2012) 383-402

12) "Search for the Higgs boson in the $\mathrm{H}->\mathrm{WW}(*)->1 \mathrm{nu} 1 \mathrm{nu}$ decay channel in pp collisions at $\sqrt{\mathrm{s}}_{\mathrm{s}}=7$ TeV with the ATLAS detector", Phys. Rev. Lett. 108, 111802 (2012)

13) "Measurement of the WZ production cross section and limits on anomalous triple gauge couplings in proton-proton collisions at $\sqrt{\mathrm{s}}_{\mathrm{s}}=7 \mathrm{TeV}$ with the ATLAS detector", Phys. Lett. B709 (2012) 341-357

14) "Kshort and Lambda production in pp interactions at $\sqrt{s}_{\mathrm{s}}=0.9$ and $7 \mathrm{TeV}$ measured with the ATLAS detector at the LHC", Phys.Rev. D85 (2012) 012001

15) "Searches for supersymmetry with the ATLAS detector using final states with two leptons and missing transverse momentum in $\sqrt{s}_{\mathrm{s}}=7 \mathrm{TeV}$ proton-proton collisions", Phys. Lett. B709 (2012) $137-$ 157

16) "Measurement of the $\mathrm{ZZ}$ production cross section and limits on anomalous neutral triple gauge couplings in proton-proton collisions at $\sqrt{s}_{\mathrm{s}}=7 \mathrm{TeV}$ with the ATLAS detector", Phys. Rev. Lett. 108 (2012) 041804

17) "Performance of the ATLAS Trigger System in 2010", Eur. Phys.J. C 72 (2012) 1849

18) "Limits on the production of the Standard Model Higgs Boson in pp collisions at $\sqrt{\mathrm{s}}_{\mathrm{s}}=7 \mathrm{TeV}$ with the ATLAS detector", Eur.Phys.J. C71 (2011) 1728

19) "Measurement of the WW cross section in $\sqrt{s}_{\mathrm{s}}=7 \mathrm{TeV}$ pp collisions with ATLAS", Phys.Rev.Lett. 107 (2011) 041802

20) "Search for high mass dilepton resonances in pp collisions at $\sqrt{s}_{\mathrm{s}}=7 \mathrm{TeV}$ with the ATLAS experiment”, Phys.Lett. B700 (2011) 163-180

21) "Search for supersymmetric particles in events with lepton pairs and large missing transverse momentum in $\sqrt{\mathrm{s}}_{\mathrm{s}}=7 \mathrm{TeV}$ proton-proton collisions with the ATLAS experiment", Eur.Phys.J. C71 (2011) 1682

22) "Search for Diphoton Events with Large Missing Transverse Energy with $36 \mathrm{pb}-1$ of $7 \mathrm{TeV}$

23) Proton-Proton Collision Data with the ATLAS Detector", Eur. Phys. J. C 71, 1744 (2011) 
24) "Search for a heavy neutral particle decaying into an electron and a muon using $1 \mathrm{fb}-1$ of ATLAS data", Eur.Phys.J.C 71 (2011) 1809

25) "Search for high-mass states with one lepton plus missing transverse momentum in proton-proton collisions at $\sqrt{s}_{\mathrm{s}}=7 \mathrm{TeV}$ with the ATLAS detector", Phys.Lett. B701 (2011) 50-69

26) "Measurement of the transverse momentum distribution of $\mathrm{Z} /$ gamma* bosons in proton-proton collisions at $\sqrt{\mathrm{s}}_{\mathrm{s}}=7 \mathrm{TeV}$ with the ATLAS detector", Phys.Lett. B705 (2011) 415-434

27) "Search for a heavy particle decaying into an electron and a muon with the ATLAS detector in $\sqrt{s}_{\mathrm{s}}=7$ TeV pp collisions at the LHC", Phys.Rev.Lett. 106 (2011) 251801

28) "Measurement of the $\mathrm{W}->$ lnu and $\mathrm{Z} /$ gamma* $->11$ production cross sections in proton-proton collisions at $\sqrt{\mathrm{s}}_{\mathrm{s}}=7 \mathrm{TeV}$ with the ATLAS detector", JHEP 1012 (2010) 060

29) "Commissioning of the ATLAS Muon Spectrometer with Cosmic Rays", Eur.Phys.J. C70 (2010) $875-916$

30) "Performance of the ATLAS Detector using First Collision Data", JHEP 1009 (2010) 056

31) "The ATLAS Simulation Infrastructure", Eur.Phys.J. C70 (2010) 823-874

32) "Charged-particle multiplicities in pp interactions at $\sqrt{s}=900 \mathrm{GeV}$ measured with the ATLAS detector at the LHC”, Phys Lett B: 688 (2010)

33) "The ATLAS Experiment at the CERN Large Hadron Collider", JINST 3 (2008) S08003

34) "A Streamlined Calibrations of the ATLAS Precision Muon Chambers for Initial LHC Running", D.S. Levin et al, accepted to Nucl. Inst. and Meth. A ( Dec 2012)

"StorNet: Co-Scheduling of End-to-End Bandwidth Reservation on Storage and Network Systems for High-Performance Data Transfers", Shawn McKee et al, IEEE High-Speed Networks, 2011.

Web link of the International Conference Notes (35) that UM physicists are primary authors http://linat05.grid.umich.edu/DOE12_UM/InternationalConf.docx

Web link of the list of the ATLAS Analysis Internal Notes (102 notes from 2009 - 2012) http://linat05.grid.umich.edu/DOE12_UM/InternalATLASNotes.docx

Web link of the talk list presented by UM physicists in conference, seminar and colloquium. http://linat05.grid.umich.edu/DOE12_UM/TalksSeminars.docx

\section{List of Rewarded Ph. D. on ATLAS Research Project}

- Hao Liu, supervisor: Jianming Qian, August 2011

"Measurement of $\mathrm{Z} \rightarrow \mathrm{e}+\mathrm{e}$ - transverse momentum distribution in proton-proton collision at $\sqrt{\mathrm{s}}_{\mathrm{s}}=7$ $\mathrm{TeV}$ in the ATLAS detector"

- John Purdham, supervisor: Rudolf Thun, September 2011

"Measurement of the Production Cross-section of $Z \rightarrow \mu+\mu$ - with ATLAS at the LHC at $\sqrt{s}_{\mathrm{s}}=7 \mathrm{TeV}$ "

- Xuefei Li, supervisor: Bing Zhou, December 2011

"Measurement of the W Boson Production Cross-section and Search for W' in Muon Channel from pp collisions at $\sqrt{s}_{\mathrm{s}}=7 \mathrm{TeV}$ with the ATLAS Detector at the LHC"

- Shannon Walch, supervisor: Jianming Qian, May 2012

"The Hunt for the Higgs Boson: the WW $\rightarrow$ lvlv final states at the ATLAS Detector" 


\title{
Top and Higgs Physics at CDF II Summary Report 2009-2011
}

\author{
D. Amidei, M. Campbell, A. Eppig, R. Edgar, D. Mietlicki, \\ T. Schwarz, G. Strycker, M. Tecchio, T. Wright \\ University of Michigan
}

\section{Introduction}

The Collider Detector at Fermilab is a general purpose device for the study of p $\bar{p}$ collisions at $\sqrt{s}=$ $1.96 \mathrm{TeV}$ at the Fermilab Tevatron. The University of Michigan high energy physics group has a 20 year investment in CDF, with contributions to silicon tracking, triggering, DAQ, operations, b-tagging, and the development of top physics from discovery tools to precision measurements. Over the threeyear period of this grant, the group has worked exclusively on the analysis of the large Tevatron data set. The individuals involved were Research Scientists Monica Tecchio and Thomas Wright, graduate students J. Clark Culley, Andrew Eppig, and Glenn Strycker, and David Mietlicki as both a student and a postdoctoral fellow.

The main focus has been on top physics. We searched for a sequential $W^{\prime}$ boson decaying to a top and bottom quark, and we made the first measurement of the spin-correlation of $t \bar{t}$ pairs as observed in the copious and reconstructable "lepton plus jets" mode. But the most important top physics result is the observation of a large forward-backward asymmetry in $t \bar{t}$ production. This result has provoked significant discussion about the NLO QCD calculation and possible new physics coupled to the top quark. The measurement is published in Ref. [1] and summarized in Section 2 below.

Using the $b$-quark identification techniques borrowed from the top analysis, we have also observed a significant mass enhancement in the two leading jets in triple $b$-tagged events. Uncovered in a search for an MSSM Higgs boson, the enhancement is consistent with the signal shape and has a $1-C L_{b} p$-value of $0.23 \%$. The result is published in Ref. [2] and summarized in Section 3 below.
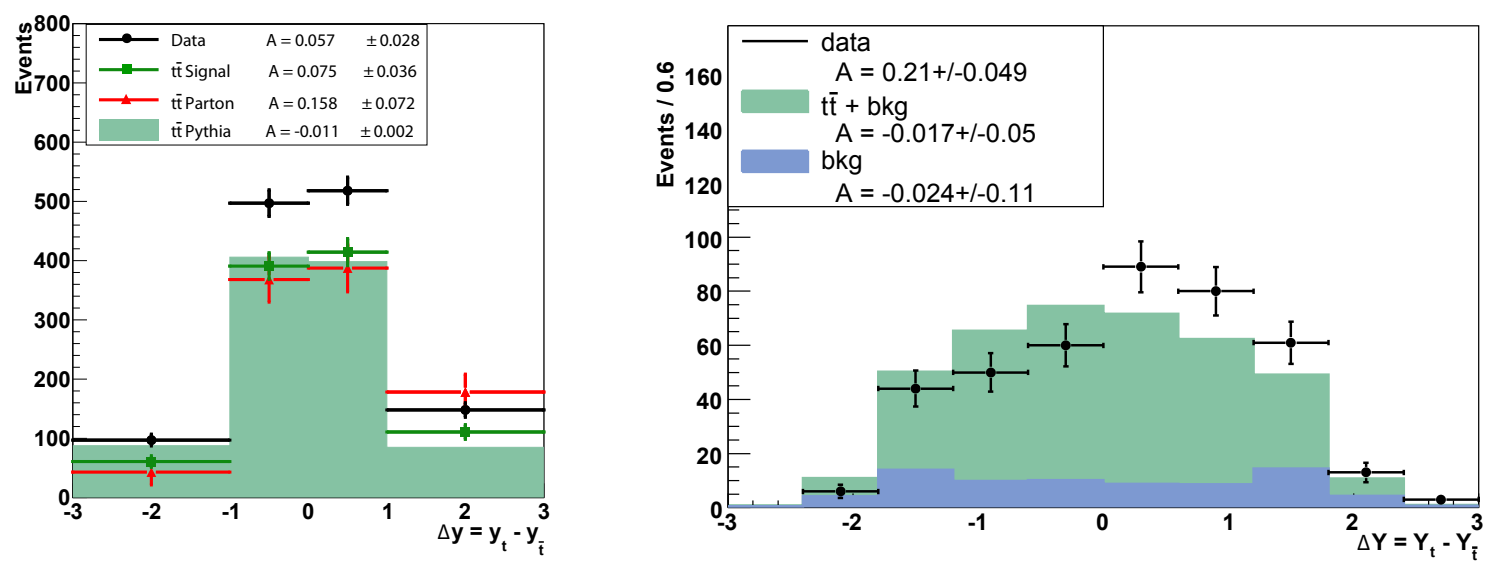

Figure 1: Left: $\Delta y$. black $=$ data, green $=$ bkgrd subtracted data, red $=$ parton level. green fill is the pythia $t \bar{t}$ model with no backgrounds, to be compared to the green markers. Right: Data level $\Delta y$ for events with $M_{t \bar{t}}>450 \mathrm{GeV} / c^{2}$ 


\section{Forward-Backward Asymmetry in $t \bar{t}$ Production}

Top quark pair production is a test of QCD at large momentum transfer. A forward-backward asymmetry is a simple way to gain insight into the production angle distribution: is it symmetric, as in the $1+\cos ^{2} \theta^{*}$ term from LO QCD, or is there an unexpected asymmetric $f$ term from some new source? The difference of the $t$ and $\bar{t}$ rapidities, $\Delta y=y_{t}-y_{\bar{t}}$, is a good approximation of the top quark rapidity in the $t \bar{t}$ rest frame and has the same sign as $\theta^{*}$. The asymmetry

$$
A_{\mathrm{FB}}=\frac{N(\Delta y>0)-N(\Delta y<0)}{N(\Delta y>0)+N(\Delta y<0)}
$$

is identical to the asymmetry in the top quark production angle in the $t \bar{t}$ rest frame.

In QCD a small asymmetry arises at order $\alpha_{s}^{3}$ from the interference of single-gluon and two-gluon processes [3, 4]. We have used the MCFM calculation and MC@NLO generator to estimate a $t \bar{t}$ frame parton level of asymmetry of $0.058 \pm 0.009$, in agreement with other calculations. Recent work [5] suggests that QED and weak interactions increase the asymmetry by a factor of 1.2 and we rescale our predictions to include that. The expected parton level inclusive asymmetry is $0.070 \pm 0.01$.

Our measurement in the $l+$ jets decay mode [1] uses $5.4 \mathrm{fb}^{-1}$ of data, a full $t \bar{t}$ reconstruction, background estimates that are well constrained by other studies, and a full correction to the parton-level. Fig.1, left, shows the CDF $\Delta y$ distribution for the raw, background subtracted, and parton level data. The parton level inclusive asymmetry at $\mathrm{CDF} A_{\mathrm{FB}}=0.158 \pm 0.074$. D0 is in good agreement with $A_{\mathrm{FB}}=0.196 \pm 0.065[6]$.

Any asymmetry is characterized by its behavior in $\cos \left(\theta^{*}\right)$ and $q^{2}$. We have performed simple differential measurements in the $l+$ jets samples, using two bins each in the proxy variables $\Delta y$ and $M_{t \bar{t}}$. $A(\Delta y)$ is calculated separately for $|\Delta y|$ greater or lesser than 1.0 and $A\left(M_{t \bar{t}}\right)$ separately for $M_{t \bar{t}}$ above and below $450 \mathrm{GeV} / c^{2}$. Results for the background-subtracted $t \bar{t}$ signal at the reconstruction level for both experiments are shown in Table 1. At CDF the strong rapidity dependence is obvious in Fig. 1, left. The significant mass dependence is seen in the high mass $\Delta y$ distribution in Fig.1. right.

These results have generated considerable interest. The CDF paper of Jan. 2011 has 231 citations and the CERN Theory group organized a Top $A_{\mathrm{FB}}$ Workshop in May 2012. There has been significant re-exaination of the small asymmetry expected in NLO QCD [5]. An NNLO calculation for the external radiative contribution $(t \bar{t}+j$ terms) suggests a decreased asymmetry at higher order [7], while the resummation approach of Ref. [3] suggests that the basic NLO result is stable to all orders. Many questions concerning both theory and observation will be resolved when the complete NNLO calculation is finally available.

More speculative discussion of the asymmetry invokes new interactions in the top sector [8]. In one group of models the gluon interferes with new axial $s$-channel objects arising from an extended strong gauge group or extra dimensions. In other models, light $t$-channel objects with flavor violating couplings create an asymmetry via the $u / d-t$ flavor change into the forward Rutherford peak. Model-building must accommodate the apparent standard model consistency in the measured cross-section and $M_{t \bar{t}}$ spectrum. Other related phenomena, such as di-jet bumps, same-sign tops, etc. lead to corollary limits or new search modes at the Tevatron and LHC. Many of these papers suggest interesting follow-up approaches to the asymmetry that are under continuing investigation and will be reported in future publications.

\section{Search for a SUSY Higgs}

In the minimal supersymmetric standard model (MSSM) with large $\tan \beta$, the production of light Higgs bosons in association with $b$-quarks can be significantly enhanced. For $\tan \beta \sim 40$ the cross section is a few picobarns [9] and the pseudoscalar Higgs boson $A$ becomes degenerate with one of the other scalars, further doubling the rate. The dominant Higgs boson decay into $b \bar{b}(\sim 90 \%)$ leads to the $3 b$ final state 
with a $\phi \rightarrow b \bar{b}$ signal enhancement in the mass of the two leading jets, $m_{12}$. Pythia + CDF simulation models for $m_{12}$ in $\mathrm{p} \overline{\mathrm{p}} \rightarrow b \phi \rightarrow b b \bar{b}$ events are shown in Fig. 2 , left.
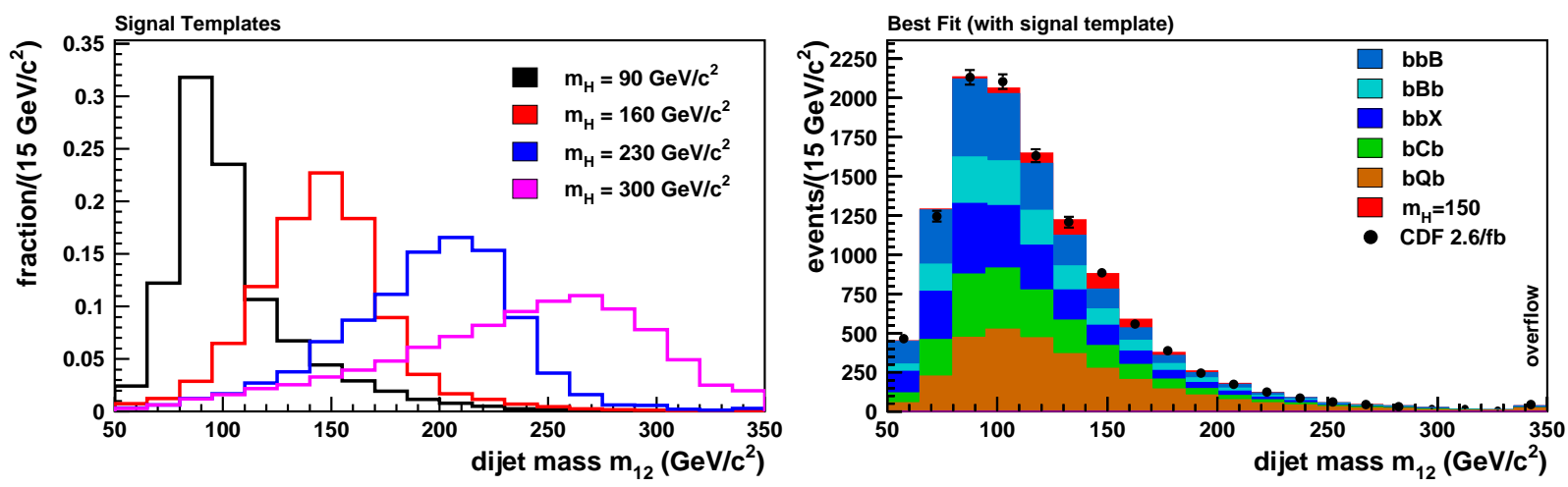

Figure 2: Left: $m_{12}$ for $H \rightarrow b \bar{b}$ as a function of $m_{H}$. Right: Best fit of background and signal model in the MSSM Higgs boson search. The Higgs signal is in red.

On our analysis [2] we use data collected with a displaced track trigger, and require at least three jets with secondary vertex tags and $E_{T}>20 \mathrm{GeV}$. The background $m_{12}$ spectrum in the triple tagged sample can therefore be estimated in the double-tag sample, once we account for the instrumental bias associated with tagging the additional jet. This depends on the flavor, $E_{T}$ rank, $\eta$, etc. of the jet, and is determined with a simulation calibrated to the data. Allowing all relevant flavor combinations gives six $m_{12}$ background shapes that together account for the QCD multi-jet contribution.

The $m_{12}$ distribution in the data is fit to a linear combination of the backgrounds and signal shapes at each mass in a $1-C L_{b}$ analysis. An example using a signal shape with $m_{\phi}=150 \mathrm{GeV} / c^{2}$ is shown in Fig. 2 (right), where the fit is seen to be robust. Fig. 3 (left) shows the 95\% CL upper limits on $\sigma(\mathrm{p} \overline{\mathrm{p}} \rightarrow \phi) \times B R(\phi \rightarrow b \bar{b})$. We observe a positive deviation of greater than $2 \sigma$ from the expectation in the region of $140-170 \mathrm{GeV} / c^{2}$. The most significant discrepancy is at $m_{\phi}=150 \mathrm{GeV} / c^{2}$, with a $1-C L_{b}$ $p$-value of $0.23 \%$. The fit for that template (back in Fig. 2), has $\chi^{2} / d o f=1.057$, with $420 \pm 130$ events assigned to the Higgs template.

Recasting this result in an MSSM model, for the $m_{h}^{\max }$ scenario with $\mu$ negative the observed limit on $\tan \beta$ is $\sim 130$ in the $m_{\phi}$ region of the excess. Limits from the LHC [10] on MSSM Higgs in the $\tau \bar{\tau}$
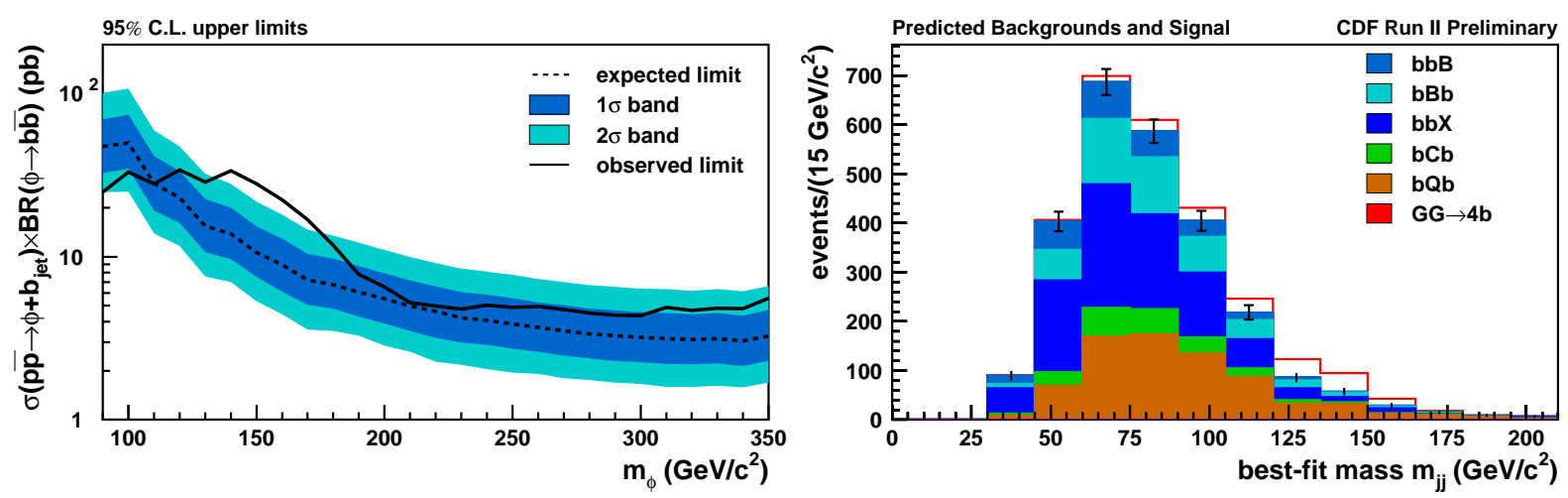

Figure 3: Left: Limits on cross section times BR from the MSSM Higgs boson search. Right: Expected background and signal in four-jet events for the color octet pair production search described in the text. The normalization and error bars on the total background correspond to a data sample of $4.5 \mathrm{fb}^{-1}$. 
channels suggest $\tan \beta<25-35$ at $95 \%$ CL, much lower than our sensitivity. The $\tan \beta$ limits in the $b \bar{b}$ decay mode are highly scenario-dependent so this may not necessarily rule out an MSSM interpretation of the excess, but it suggests that, if the excess is real, it is important to explore other models.

\section{Publications}

1. T. Aaltonen et al., The CDF Collaboration, "Search for the Production of Narrow $t \bar{b}$ Resonances

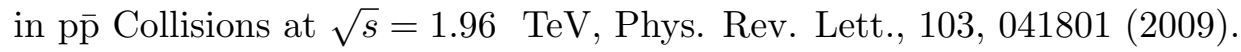

2. T. Aaltonen et al., The CDF Collaboration, "Measurement of $t \bar{t}$ Spin Correlation in p $\bar{p}$ Collisions Using the CDF II Detector at the Tevatron", Phys. Rev. D83, 031104(R) (2011).

3. T. Aaltonen et al., The CDF Collaboration, "Evidence for a Mass Dependent Forward-Backward Asymmetry in Top Quark Pair Production," Phys. Rev. D83:112003, (2011).

4. T. Aaltonen et al., The CDF Collaboration, "Search for Higgs Bosons Produced in Association with $b$ Quarks", Phys. Rev. D 85, 032005 (2012).

\section{$5 \quad$ Ph.D Theses}

1. J. Clark Cully, "Search for $W^{\prime} \rightarrow t \bar{b}$ in p p Collisions at $\sqrt{s}=1.96 \mathrm{TeV}$ ", University of Michigan, Dec. 2008.

2. David Mietlicki, "Measurement of $t \bar{t}$ Helicity Fractions and Spin Correlation in p $\bar{p}$ Collisions at $\sqrt{s}=1.96 \mathrm{TeV} "$, University of Michigan, Aug. 2010.

3. Glenn Strycker, "Measurement of the Inclusive Forward-Backward $t \bar{t}$ Production Asymmetry and Its Rapidity Dependence", University of Michigan, Sept. 2010.

4. Andrew Eppig, "Forward-Backward Asymmetry at High Mass in $t \bar{t}$ Production in p $\bar{p}$ Collisions at $\sqrt{s}=1.96 \mathrm{TeV} "$, University of Michigan, April, 2011.

\section{Conference Talks}

1. M. Tecchio, "Mass Dependence of the Forward-Backward Asymmetry in Top Pair Production", 2009 DPF Detroit, Jul 2009

2. D. Mietlicki, "Measurement of Top Pair Spin Correlation Using CDF Run II Lepton+Jets Data", 2010 APS April Meeting, February 2010.

3. T. Wright, Higgs Searches at the Tevatron, Conference on LHC First Data, Univ. of Michigan, December 2010.

4. D. Amidei, "A Mass Dependent Asymmetry in Top Quark Pair Production",Fermilab Joint ExperimentTheoretical Seminar, Jan. 2011.

5. D. Amidei, "A Forward-Backward Asymmetry in Top Quark Pair Production", les Rencontres de Physique de la Vallee dAoste, LaThuile, Italy, Mar. 2011.

6. T. Wright, "Search for Higgs Bosons Produced in Association with b-Quarks at CDF", Fermilab Joint Experiment-Theoretical Seminar, July 2011.

7. T. Wright, "Tevatron Non-Standard Model Higgs Searches", SUSY 2011, Fermilab, August 2011. 
8. T. Wright, "SUSY Higgs Searches in the bbb Final State", SUSY 2011, Fermilab, August 2011.

9. D. Mietlicki, "Top Quark Production at the Tevatron", Physics In Collision 2011, September 2011.

10. D. Mietlicki, "Measurements of Top Quark Properties at the Tevatron", 2012 Moriond QCD, March 2012.

11. D. Mietlicki, "The Top Pair Forward-Backward Asymmetry with the Full CDF Dataset", Fermilab Joint Experiment-Theoretical Seminar, March, 2012.

12. D. Amidei, "Forward-Backward Asymmetry in Top Quark Pair Production", Top Physics from Charge Asymmetry to the Boosted Regime, CERN, May. 2012.

\section{Conclusion}

We have searched for $W^{\prime}$ decays to top and bottom quarks, and we have measured the spin structure of $t \bar{t}$ pairs produced in single gluon annihilation. We present evidence for a significant mass dependent asymmetry in $t \bar{t}$ production. If the asymmetry is real it could be evidence for new interactions in the top sector or unexpected behavior of QCD at higher order. We also present evidence for a significant mass enhancement in $b \bar{b}$ production in association with another $b$, which has some similarities to a MSSM Higgs boson with large $\tan \beta$ or models for color octets.

\section{CDF References}

[1] T. Aaltonen et al. (CDF Collaboration), Phys. Rev. D 83, 112003 (2011).

[2] T. Aaltonen et al. (CDF Collaboration), Phys. Rev. D 85, 032005 (2012).

[3] L. G. Almeida, G. F. Sterman and W. Vogelsang, Phys. Rev. D 78, 014008 (2008).

[4] O. Antunano, J. H. Kuhn, and G. V. Rodrigo, Phys. Rev. D 77, 014003 (2008); M. T. Bowen, S. D. Ellis, and D. Rainwater, Phys. Rev. D 73, 014008 (2006).

[5] W. Hollik, D. Pagani, arXiv:1107.2606; J. H. Kuhn, G. Rodrigo, arXiv:1109.6830.

[6] V. M. Abasov et al. (D0 Collaboration) Phys. Rev. D 84, 112005 (2011.)

[7] S. Dittmaier,P. Uwer, and S. Weinzer, Nucl. Phys. B, Proc. Suppl. 183, 196 (2008).

[8] Good comprehensive reviews are M. Gresham, I. W. Kim, K. Zurek, arXiv:1102.0018 and Q.-H. Cao, D. McKeen, J. Rosner, G. Shaughnessy, and C. Wagner, Phys. Rev. D 81, 114004 (2010).

[9] S. Dawson, C. B. Jackson, L. Reina and D. Wackeroth, Mod. Phys. Lett. A 21, 89 (2006) [arXiv:hep$\mathrm{ph} / 0508293]$.

[10] S. Chatrchyan et al. [ CMS Collaboration ], Phys. Rev. Lett. 106, 231801 (2011). [arXiv:1104.1619 [hep-ex]]. 


\title{
Summary report of the Michigan DØ project for FY 2009-2012
}

\author{
K. Herner, J. Qian, A. Wilson, C. Xu, J. Yu, B. Zhou, J. Zhu \\ Department of Physics, University of Michigan, Ann Arbor, Michigan 48109
}

August 15, 2012

\section{Introduction}

This brief report summarizes our activities and achievements in the DØ experiment at the Fermilab Tevatron $p \bar{p}$ collider over the past three-year funding cycle. The Michigan D $\varnothing$ group has had a long productive participation in the experiment. By all measures, the Tevatron has been a success, exceeding its design goals in both instantaneous and integrated luminosity. It delivered a total integrated luminosity of $\sim 12 \mathrm{fb}^{-1}$ to the $\mathrm{D} \varnothing$ experiment before its de-commissioning on September 30, 2011. Though the energy frontier is now at the LHC at CERN, the nature of $p \bar{p}$ collisions keeps Tevatron results competitive in some selected topics. Among them are searches for a low mass Standard Model (SM) Higgs boson in its decay to a pair of $b$-quarks, our main activity over the past three years. In addition, we have carried out a search for supersymmetry in $Z \gamma$ events with a large transverse momentum imbalance $\left(\not_{T}\right)$ and performed a precise measurement of the effective weak mixing angle $\sin ^{2} \theta_{\text {eff }}^{\text {lept }}$.

\section{Search for a low-mass Standard Model Higgs boson}

Searching for a light SM Higgs boson at the Tevatron has been our main analysis activity in DØ over the past three years. Our group, though small, has taken a leading role in the Higgs searches in D $\varnothing$. Postdoc Ken Herner first served as a convener for the low-mass Higgs working group and is now a convener for the overall Higgs working group.

For the mass range above the LEP limit of $114.4 \mathrm{GeV}[1]$ and below approximately $135 \mathrm{GeV}$, the Higgs boson is predicted to predominantly decay to $b \bar{b}$. Without a leptonic final state, a light Higgs in the dominant $g g \rightarrow H$ and $q q \rightarrow q q H$ production processes is obscured by backgrounds at the Tevatron and in fact is even more difficult to observe at the LHC. Thus, one of the most promising channels at the Tevatron is the Higgs production in association with a vector boson, $W$ or $Z$. When a $W$ or $Z$ boson decays leptonically, the resulting charged electron or muon allows for easy triggering. Together with the neutrino in the case of $W$ decay, they provide handles to discriminate against the otherwise overwhelming multijet background.

We have been participating in all aspects of the $W H \rightarrow \ell \nu b \bar{b}$ analysis from the analysis of the initial $1 \mathrm{fb}^{-1}$ dataset to the current analysis with the full $9.7 \mathrm{fb}^{-1}$ dataset. Our effort spans from trigger improvement, lepton identification, jet energy resolution, background modeling, systematic uncertainties and finally the signal-background separations. We increased muon acceptance by $15 \%$ through the improvement of Monte Carlo modeling of the trigger in the high pseduorapidity region. We studied lepton identification requirements which led to $\sim 10 \%$ efficiency gain for both electrons and muons. We worked on the dijet mass resolution and the optimization of the $b$-jet tagging. These improvements combined have led to significant enhancement of the intrinsic sensitivity of the analysis. We developed multivariate techniques to improve signal and background separation of the $W H$ analysis, starting with the matrix-element (ME) method and evolving to the current the boosted decision tree (BDT) [2]. Apart from Herner, other group members participated in this analysis are faculty Jianming Qian and student Chun Xu (graduated). 
We have also performed a $Z H$ search using $9.7 \mathrm{fb}^{-1}$ of data focusing on the $Z H \rightarrow \mu \mu b \bar{b}$ final state. Compared with previous analysis, we used a two-step multivariate analysis strategy based on random forest discriminants to further improve the separation of signal from background. We are responsible for the BDT training and the statistical interpretation and combination of $\ell \ell b \bar{b}$ results. This analysis is done by student Jiaming Yu supervised by Prof. Junjie Zhu.

The results from $W H \rightarrow \ell \nu b \bar{b}$ and $Z H \rightarrow \mu \mu b \bar{b}$ searches are combined with searches in other final states, most notably $Z H \rightarrow e e b \bar{b}, \nu \bar{\nu} b \bar{b}$ for the low mass region. Figure 1 (left) shows the expected and observed $95 \%$ confidence level (CL) cross section limits as a ratio to the SM expectation from the combination. In the low mass region, small excesses in the data over the background-only hypothesis led to weaker observed limits than expectations. At $m_{H}=120 \mathrm{GeV}$, the observed $p$-value of a background-only hypothesis is $2.8 \%$, corresponding to $1.9 \sigma$. Combination results with CDF are shown in Fig. 1 (right). The broad excess at low mass remains with a $p$-value of background fluctuation of $0.15 \%(3.0 \sigma)$. These results are consistent with the existence of a low-mass SM Higgs boson and with the observation of a new particle recently announced by ATLAS and CMS experiments at the LHC $[3,4]$.
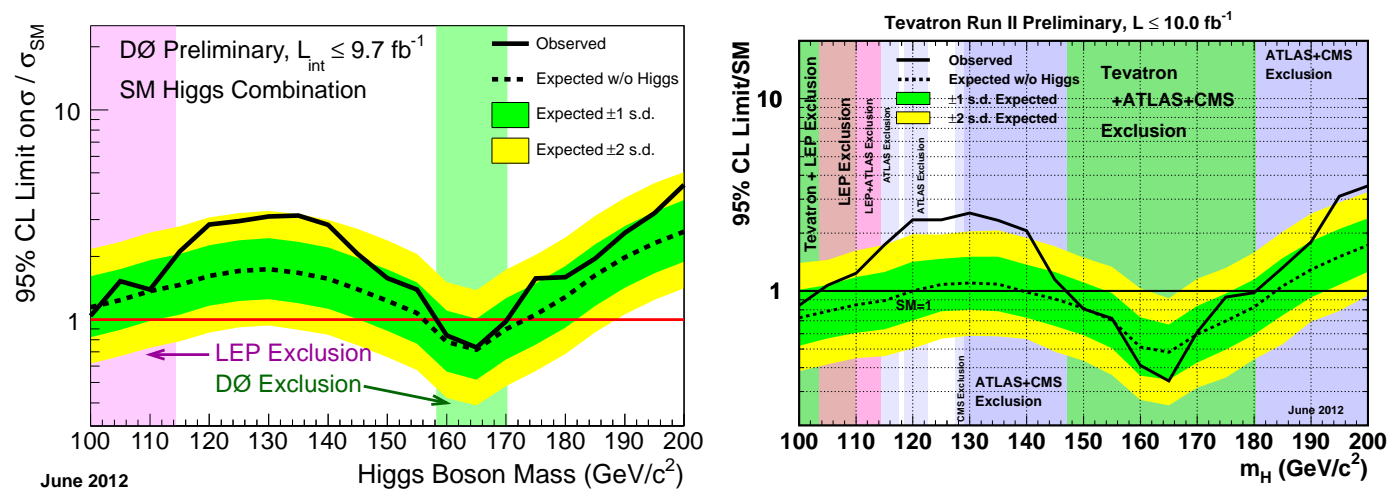

Figure 1: Expected and observed 95\% CL cross section upper limit relative to the Standard Model expectation as a function of the Higgs boson mass combining all searches: D $\varnothing$ combination (left) and Tevatron combination (right).

\section{Search for supersymmetry in $Z \gamma+Z_{T}$ events}

We had a small effort in the search for events with a $\mathrm{Z}$ boson, a photon $(\gamma)$ and large missing energy, as expected from supersymmetric models with gauge mediated symmetry breaking (GMSB) [5]. This analysis was the Ph.D. thesis project of our student Alan Wilson supervised by Prof. Bing Zhou. Alan worked on the ATLAS project since 2004 and made major contributions to ATLAS endcap muon detector integration, installation and commissioning during 2004-2006. He switched to DØ to work on his thesis after the LHC magnet incident in fall 2008.

In GMSB models, the gravitino $\tilde{G}$ is the lightest supersymmetric particle (LSP) while the lightest neutralino $\left.\left(\tilde{\chi}_{1}^{0}\right)\right)$ is the next lightest supersymmetric particle (NLSP) for much of the parameter space. Our analysis is focused on the supersymmetry parameter region with a Higgsino-like NLSP. In this scenario, the NLSP neutralino decays $\tilde{\chi}_{1}^{0} \rightarrow \tilde{G}+Z, \tilde{G}+\gamma$ have significant branching ratios. $\tilde{\chi}_{1}^{0}$ can be pair produced either directly or indirectly from decays of other supersymmetric particles. We look for events with one $\tilde{\chi}_{1}^{0}$ decaying to $\tilde{G}+Z$ and the other to $\tilde{G}+\gamma: \tilde{\chi}_{1}^{0} \tilde{\chi}_{1}^{0} \rightarrow \tilde{G} Z+\tilde{G} \gamma \rightarrow Z \gamma+\not_{T}$. The gravitinos as the LSP are stable and weakly interacting. They escape undetected leading to significant $\not_{T}$. Thus pair production of $\tilde{\chi}_{1}^{0}$ will give rise to $Z \gamma+\not_{T}$ events. No excess beyond 
background expectation is observed. Cross section limits on the $Z \gamma+\not t_{T}$ production are set (see Fig. 2). The results of the search have been submitted for publication (arXiv:1203.5311).

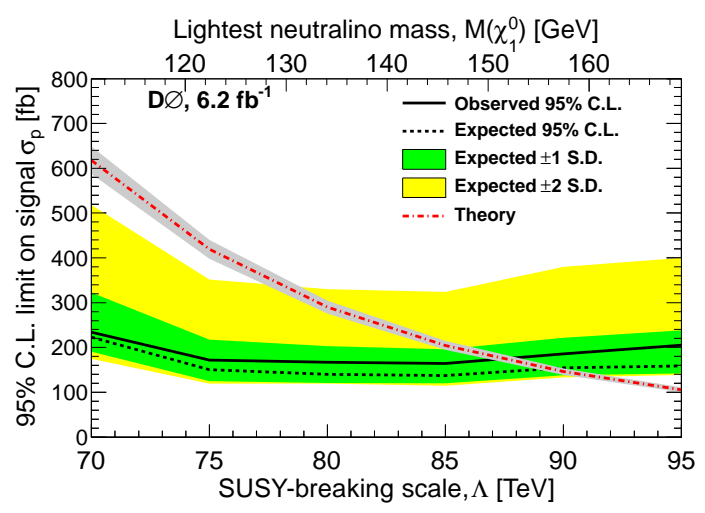

Figure 2: Limit on the cross section for $Z \gamma+\not_{T}$ production as a function of supersymmetry breaking scale $\Lambda$ at $95 \%$ CL.

\section{Electroweak measurement}

Since joining Michigan faculty in 2010 to primarily work on the ATLAS experiment, Zhu continued to work on the measurement of $Z / \gamma^{*}$ forward-background charge asymmetry in D $\varnothing$ to finish it up for publication. From the mass dependence of the asymmetry determined from a dataset of $5.0 \mathrm{fb}^{-1}$ of integrated luminosity, the effective weak mixing angle is measured to be $0.2309 \pm 0.0008($ stat. $) \pm$ 0.0006 (syst.). We also presented the most precise direct measurement of the vector and axial-vector couplings of $u$ and $d$ quarks to the $Z$ boson. Since the physics process we used involves predominantly the first generation light quarks, our results are complementary to other results from the LEP, SLD and HERA experiments [6, 7]. The results of this analysis have been published (Phys. Rev. D84, 012007 (2011)).

\section{References}

[1] ALEPH, DELPHI, L3 and OPAL Collaborations, LEP electroweak working group, http://lepewwg.web.cern.ch/LEPEWWG/

[2] L. Breiman et al., Classification and Regression Trees (Wadsworth, Stamford, 1984).

[3] ATLAS Collaboration, Observation of a new particle in the search for the Standard Model Higgs boson with the ATLAS detector at the LHC, arXiv:1207.7214, submitted to Phys. Lett. B

[4] CMS Collaboration, Observation of a new boson at a mass of 125 GeV with the CMS experiment at the LHC, arXiv:1207.7235, submitted to Phys. Lett. B.

[5] See G.F. Giudice and R. Rattazzi, Phys. Rept. 322, 419 (1999) and references therein.

[6] LEP ElectroweakWorking Group, Precision electroweak measurements on the $Z$ resonance, Phys. Rept. 427, 257 (2006).

[7] NuTeV Collaboration, A precise determination of electroweak parameters in neutrino nucleon scattering, Phys. Rev. Lett. 88, 091802 (2002), Erratum-ibid 90, 239902 (2003). 


\section{Completed Ph.D Thesis}

- Alan Wilson (2011)

Search for a Supersymmetric Signature with the $Z \gamma$ Plus Missing Transverse Energy Final State Using the DØ Detector

- Chun Xu (2010)

Search for the Standard Model Higgs Boson in Asscoiated Production with $W$ Boson at the Tevatron

\section{Publication with major Michigan contributions}

- CDF and DØ Collaborations, Evidence for a particle produced in association with weak bosons and decaying to a bottom-antibottom quark pair in Higgs boson searches at the Tevatron, arXiv:1207.6436, to appear in Phys. Rev. Lett.

- DØ Collaboration, Combined search for the standard model Higgs boson decaying to $b \bar{b}$ using the DØ Run II data, arXiv:1207.6631, submitted to Phys. Rev. Lett.

- DØ Collaboration, Search for the standard model Higgs boson in associated WH production in $9.7 \mathrm{fb}^{-1}$ of $p \bar{p}$ collisions with the DØ detector, arXiv:1208.0653, submitted to Phys. Rev. Lett.

- DØ Collaboration, Search for the standard model Higgs boson in $Z H \rightarrow \ell^{+} \ell^{-} b b$ production with the DØ detector in $9.7 \mathrm{fb}^{-1}$ of pp collisions at $\sqrt{s}=1.96 \mathrm{TeV}$, arXiv:1207.5819, submitted to Phys. Rev. Lett.

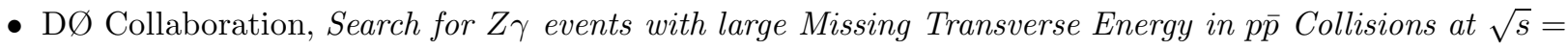
$1.96 \mathrm{TeV}$, arXiv:1203.5311, submitted to Phys. Rev. Lett.

- DØ Collaboration, Search for WH associated production in p $\bar{p}$ collisions at $\sqrt{s}=1.96$ TeV, arXiv:1203.1082, to appear in Phys. Rev. D.

- DØ Collaboration, Search for WH associated production in $5.3 \mathrm{fb}^{-1}$ of $p \bar{p}$ collisions at the Fermilab Tevatron, Phys. Lett. B 698, 6 (2011).

- DØ Collaboration, Measurement of $\sin ^{2} \theta_{\text {eff }}^{\text {lept }}$ and Z-light quark couplings using the forward-backward charge asymmetry in $p \bar{p} \rightarrow Z / \gamma^{*} \rightarrow$ ee events with $L=5.0 \mathrm{fb}^{-1}$ at $\sqrt{s}=1.96 \mathrm{TeV}$, Phys. Rev. D 84, 012007 (2011).

- DØ Collaboration, Search for Associated $W$ and Higgs Boson Produciton in p p Collisions at $\sqrt{s}=$ 1.96 TeV, Phys. Rev. Lett. 102, 051803 (2009).

\section{Conference Presentations}

- K. Herner, Combination of DØ Searches for the SM Higgs Boson, 36th International Conference on High Energy Physics (ICHEP), Melbourne, Australia, July 2012.

- K. Herner, WZ/ZZ measurements and evidence for diboson processes in b-tagged final states, Joint Theoretical-Experimental Physics Seminar (Wine and Cheese Seminar), Fermilab, December 2011.

- J. Zhu, Latest electroweak results from the Tevatron experiments, Physics at the LHC, Perugia, Italy, June 2011.

- K. Herner, Low-mass standard model Higgs searches at the Tevatron, Aspen 2011 - New Data from the Energy Frontier, Aspen, CO, February 2011.

- J. Zhu, Physics of $W$ and $Z$ bosons, RIKEN BNL Research Center workshop, Brookhaven, New York, June 2010.

- J. Qian, Selected Results from DØ, Planck - From the Planck Scale to the Electroweak Scale, CERN, May 2010.

- K. Herner, Standard Model Higgs searches at the Tevatron, XVIII International Workshop on DeepInelastic Scattering and Related Subjects, Firenze, Italy, April 2010. 


\section{KOTO: M. Campbell, M. Tecchio}

\section{Report for the period 2009-2012}

During the previous grant period we have been working on the J-PARC $\mathrm{K}_{\mathrm{L}}$ E14 (KOTO) experiment with the goal to discover and measure the rate of the rare decay $K_{L} \rightarrow \pi^{0} v \bar{v}$. This $\mathrm{CP}$-violating flavor changing neutral current decay proceeds through second-order weak interactions. Other, as yet undiscovered particles, which can mediate the decay could provide an enhancement to the branching ratio, which in the Standard Model predicted to be about $(2.8 \pm 0.4) \times 10^{-11} 1$. The experiment is expected to observe 100 events at the Standard Model branching ratio for a 10\% measurement. The experiment is a follow-up to E391a at KEK and has been approved as experiment E14 at J-PARC. The main barrel vacuum vessel, the charged veto detectors, and the main barrel photon veto system will be reused from E391a. The main calorimeter has been replaced with Cesium Iodide crystals that are both smaller to provide improved shower reconstruction and longer to prevent energy leakage out of the back of the calorimeter. New trigger and data acquisitions electronics will be used.

A construction grant separate from the DoE base funding supports the US institutions (University of Michigan, University of Chicago and Arizona State University) working on the experiment and provides the Cesium Iodide calorimeter and readout. The CsI crystals were previously used at the $\mathrm{KTeV}$ experiment at Fermilab. New readout electronics trigger and data acquisition are being developed and constructed to complete the calorimeter system. The construction grant provided \$600,000 in FY09 (9/1/2009 through 8/31/2010) and \$550,000 in FY10 (9/1/2010 through 8/31/2011).

Since the start of the construction grant (September 1, 2009) we have been working on designing, building and testing prototypes for the trigger and data acquisition system for the KOTO experiment. Production versions of the ADC board, Level 1 trigger board, Level 2 trigger board and fanout board have been made. In January 2010 an integration test at the Tohoku University electron beam was done to verify that all of the components worked together. A testrun at J-PARC was performed in fall 2010 when the properties of the neutral beam and performance of the detector and electronics was measured.

The efforts at University of Michigan have been focused on the design and construction of the trigger and data acquisition system. At the time of the E14 proposal, our best estimates for the level 1 trigger rates were based on extrapolations from E391a data. A full KOTO simulation has become available in the meantime and has been used to calculate expected level 1 trigger rates of $120 \mathrm{kHz}$ for a total calorimeter energy threshold of $300 \mathrm{MeV}$ and some minimal charged veto and neutral veto requirements. This also assumes $8 \cdot 10^{6} \mathrm{~K}_{\mathrm{L}}$ per spill, which is the final Step 1 production rate for $2 \cdot 10^{14}$ protons of $30 \mathrm{GeV}$.

Over the last two years, we completed the backbone of the K0TO data acquisition system. A block diagram of the system is shown in Figure 1. It consists of frontend ADC 6U VME crates, two Level 1 and Level 2 Trigger 9U VME crates, a 9U VME Fanout crate and a cluster of PCs for event reconstruction and data storage. 


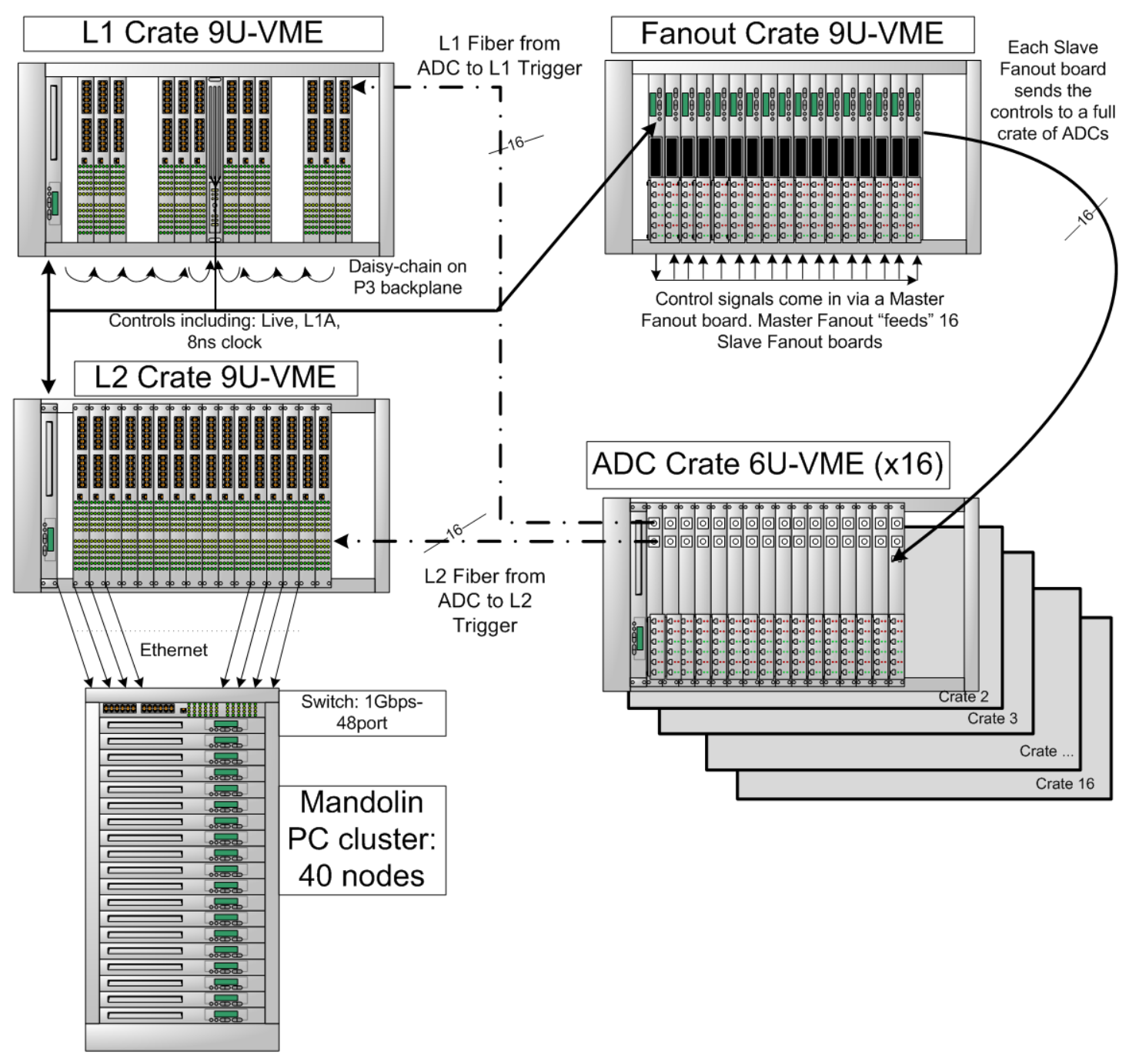

Figure 1: Block Diagram of the KOTO Data Acquisition System

The sixteen frontend crates, used to readout both the calorimeter and the veto detectors, have up to sixteen $125 \mathrm{MHz}$ ADC boards each. Each ADC module can shape and digitize the PMT's pulse from 16 detector channels. The whole readout consists of about $2800 \mathrm{CsI}$ calorimeter channels and about 1000 upstream, barrel and beam collar vetoes. We have received the 230 ADC boards, needed for reading out all of the CsI channels and most of the vetos, and delivered them to Japan housed in 6U VME crates, along with the cables carrying the analog signals from the detector.

Each ADC board has three connections to the rest of the DAQ. The first is used to distribute the main sampling clock, the Level 1 Trigger accept (L1A) decision and the $\mathrm{t}_{0}$ (or LIVE) for the whole readout. These signals are generated by a Master Control module, sitting in the Level 1 Trigger crate. A fourth control signal is used to communicate back to the Master Control module whether any frontend ADC module in the system has an error condition that prevents it from providing valid data to the readout chain. These four control signals are distributed via a Fanout module, specifically designed to provide tightly matched routing copies of each signals to multiple boards. Each Fanout module can service a full frontend crate. We have fabricated and tested 20 Fanout modules and 15 have been delivered to Japan in a 9U VME crate, together with the control signal cables. 
The second connection is the L1 fiber carrying the digitized energy sum over the 16 frontend channels. This $50 \mu \mathrm{m}$ multi-mode fiber, driven by a $2.5 \mathrm{~Gb} / \mathrm{s}$ optical transceiver, connects up to sixteen ADC channels to a single Level 1Trigger board in the Level 1 Crate. Here the fiber information is de-serialized and aligned with respect to the system $t_{0}$ before being passed in daisy-chain fashion to an adjacent Level 1 Trigger board in the Level 1 crate. The daisy-chain connection, implemented in a custom-made backplane, allows for the total energy over the entire CsI calorimeter to be computed inside the Master Control module so that a first level trigger decision can be made upon comparing the total energy to a predetermined threshold. A Level 1 trigger results in the full readout of 48 ADC samples for each channel.

The third and final connection is the Level 2 fiber, carrying the digitized energy for every channel needed to reconstruct the Gaussian shaped pulse of each PMT for an event accepted by the Level 1 Trigger. The Level 2 fiber uses the fast optical link and can accommodate Level 1 trigger rates up to $167 \mathrm{kHz}$ even with no pedestal subtraction algorithm. The Level 2 Trigger board shares the same hardware as the Level 1Trigger board and, in the present implementation of the trigger, acts mostly as a readout board. The main difference between the two boards is the installation of two on-board 2 Gb DRAM memories, plus a controlling FPGA, and a 1Gb/sec Ethernet port. This port is the main conduit for the event readout from the trigger to the event reconstruction and data storage. The memories are chosen to match the amount of data we can read out via the Ethernet port in the 3.3s between the accelerator spills. While one memory is read out, the other is used to store the energies received during the $0.7 \mathrm{~s}$ of protons on target. This dual memory configuration allow for maximum event rates up to $14 \mathrm{kHz}$. We have fabricated and tested 40 Trigger boards, of which 18 have been delivered to Japan in two 9U VME crates.

Data recorded by each Level 2 Trigger board are sent via a 48-port switch to a 41-node computer cluster which we named Mandolin. Each processor in the cluster collects partial events from each of the sixteen Level 2 trigger boards and does offline event reconstruction and data writing to hard disk in parallel across multiple triggers. The cluster is configured with 1 master and 40 server nodes. Each node has two dual-core 2.6 GHz AMD Opteron processors, a $250 \mathrm{~GB}$ system disk, and a 750 GB data disk, for a total of 30 TB data storage. Mandolin has already been delivered to Japan and was used to collect and store the data from the December 2010 testrun.

We have continued to examine possible low level background processes. One possible background is the kaon decays via $K_{L} \rightarrow \pi^{+} \mu^{-} \bar{\nu}$ where the muon and pion form a $\pi$ - $\mu$ atom, which has a $1.05 \times 10^{-7}$ branching ratio. The $\pi$ - $\mu$ atom could then decay through muon capture to a final state of $\pi^{0} v$, giving an identical final state as the signal. This decay chain goes through two sequential first order weak decays, while the signal proceeds through a single second order weak decay. Graduate student Jia Xu performed a detailed calculation of the $\pi$ - $\mu$ atom muon capture and estimates the lifetime to be on the order of 1 second, well above the lifetime of $10^{-5}$ seconds for this process to be a background.

In 2010, two major runs took place in J-PARC. The first was a beam-survey in January through February 2010, with the goal of characterizing the physical dimensions and particle composition of the $\mathrm{K}_{\mathrm{L}}$ beamline as described above. At the same time a CsI test was being performed at Tohoku University in Sendai. The second run took place in October and November 2010. This was a partial CsI calorimeter commissioning run, for which photon and electron data were taken for about 1200 CsI channels using a prototype version of the DAQ electronics. The results of reconstructing $K_{L} \rightarrow \pi^{+} \pi^{-} \pi^{0}$ is shown in Figure 2, and this verified the beam shape and intensity. Monica Tecchio, Shumin Li and Jon Ameel participated in the testrun. 


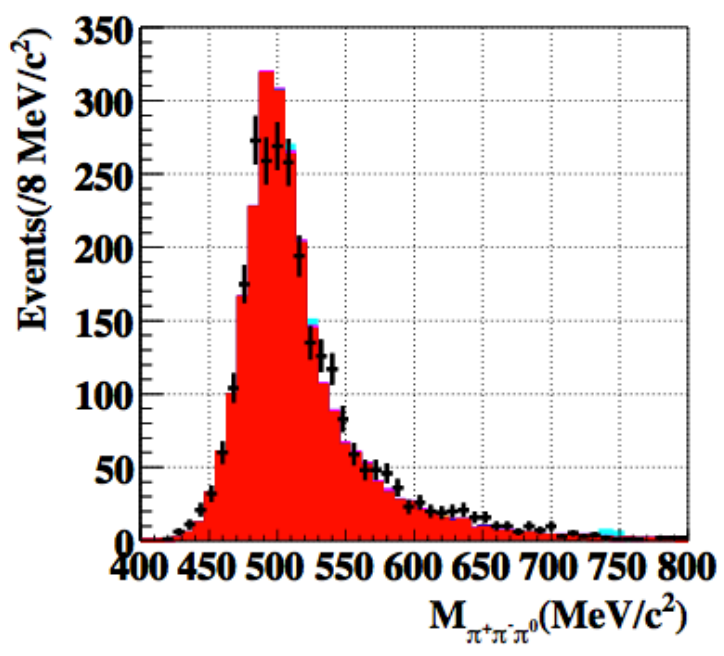

Figure 2: The invariant mass distribution of $K_{L} \rightarrow \pi^{+} \pi^{-} \pi^{0}$ reconstruction.

During the CsI commissioning run, a $0.7 \mathrm{~T}$ spectrometer upstream of the calorimeter was used to measure the momentum of electron and pions. Energy calibration constants for each crystal obtained using cosmic data were validated by using the momentum of electrons measured in the spectrometer. The energy response of the calorimeter was also studied with single $\pi^{0}$ in special runs with an aluminum target inserted in the beamline upstream of the calorimeter and by forcing events with two reconstructed photons to have a common vertex at the target. For data triggered using the total calorimeter energy, we could reconstruct events with six photons and reconstructed $K_{L} \rightarrow \pi^{0} \pi^{0} \pi^{0}$ events.

The Great East Japan Earthquake has delayed this experiment by about one year. A full calorimeter engineering, scheduled for April 2011, took place in March and June 2012. Several people from the University of Michigan participated in and contributed to this run. Professor Myron Campbell was at J-PARC for 10 days and worked on installing the electronics and setup a teststand for the main barrel modules. Research Scientist Monica Tecchio was at J-PARC for five weeks and installed and verified the DAQ system. Graduate Student Jia Xu was at J-PARC for nine weeks and successfully brought the DAQ system and trigger system up to full performance of 10,000 events per spill. Jia's Ph.D. thesis will be on the Grossman-Nir bound which we expect to reach by June 2013. Undergraduate Celeste Carruth stayed at J-PARC for 10 weeks and worked on a trigger algorithm to reduce the trigger rate due to the dominate decay modes of $K_{L} \rightarrow \pi^{0} \pi^{0} \pi^{0}$ and $K_{L} \rightarrow \pi^{0} \pi^{0}$. This will form the basis of her senior thesis. Undergraduate Jennifer Miller was at J-PARC for ten weeks. Jennifer wrote a program to measure and track the pedestals for all ADC modules and store the results in a run conditions data base.

\section{Publications}

2009 DPF Detroit, “The KOTO Experiment”, July 2009.

Flavor Physics and CP Violation 2010, “ $K \rightarrow \pi \nu \bar{\nu}$ Experiments”, Torino, May 2010.

Technology and Instrumentation in Particle Physics 2011, "The Data Acquistion System for the KOTO Detector”, Chicago, June 2011 


\section{References}

${ }^{1}$ A. J. Buras, M. Gorbahn, U. Haisch, and U. Nierste, hep-ph/0603079; Phys. Rev. Lett. 95, 261805 (2005). 
Task I Final Report (2009 - 2012)

\section{Summary}

This task supports a research program that addresses some of the most pressing questions in astrophysics and cosmology: What is the nature of the mysterious "dark energy" that appears to be responsible for the accelerating expansion of the universe? How have the largest structures in the universe formed and evolved? What instrumentation and software techniques are needed to carry out the next generation of precision observations? Over the most recent funding period, the University of Michigan Dark Energy group has consisted of faculty members David Gerdes, Wolfgang Lorenzon, Tim McKay, and Gregory Tarlé; Associate Research Scientist Michael Schubnell; postdoctoral fellow Jeeseon Song; graduate students Jiangang Hao, Tomasz Biesiadzinski, and Adam Sypniewski; and a number of undergraduate students. Over the course of this funding period our major projects have included the Joint Dark Energy Mission (JDEM), the Dark Energy Survey (DES), and the upcoming BigBOSS project. Our state-of-the-art nearinfrared detector laboratory supports our work on the next generation of experiments. We discuss our contributions to these efforts below. A complete list of publications arising from our work is given at the end.

\section{Dark Energy Survey}

The Dark Energy Survey (DES), scheduled to begin in early 2013, is an optical survey of 5000 square degrees of the southern sky being carried out by an international team of researchers at Cerro Tololo Inter-American Observatory in Chile. Using the new Dark Energy Camera at the prime focus of the 4-meter Blanco telescope, DES will obtain multiband images of over 300 million galaxies, and will discover about 2000 Type Ia supernovae - a data sample of unprecedented depth and richness. Using these data, we will carry out measurements of the dark energy equation of state using four independent, complementary techniques: weak gravitational lensing, Type Ia supernovae, galaxygalaxy correlations, and galaxy clusters. The UM group's work has particularly emphasized the latter technique.

The Dark Energy Camera was designed and built over an 8-year period beginning in 2004. UM's contributions to this instrument have included:

- Optical design of the camera (by UM faculty member Rebecca Bernstein, now at UCSD)

- Development of specifications for the filters

- Design, construction, testing, and commissioning of the filter-changer mechanism

- Development of an alignment-monitoring system for the camera

In addition, we contributed observing time to the PreCam survey, a pre-DES calibration effort using UM's 0.9-meter Curtis-Schmidt telescope at CTIO. Faculty member Tim McKay co-led the Galaxy Cluster working group, and Gus Evrard co-leads the 
Simulation working group. Gerdes chairs the Membership Committee, while Tarlé serves on the Publication Committee.

We have prepared for the arrival of DES survey data through a number of efforts aimed at strengthening the connection between observables (e.g. galaxy cluster optical richness, Sunyaev-Zel'dovich signals), and quantities that can be predicted by theory (halo masses, underlying cosmological parameters). The UM group leads an ambitious effort within DES called the Cluster-Finder Comparison Project, the first large-scale, systematic comparison between over half a dozen different optical galaxy cluster finders. In addition, we are playing a leading role in DES's Blind Cosmology Challenge, in which DES science analyses are performed on simulated catalogs with unknown (to the participants) cosmological parameters. It is crucial to carry out such demonstration projects before the first survey data begin to arrive in order to refine our simulations, establish a baseline understanding of our analysis methods, and uncover possible systematics.

Most measurements DES will require knowledge of the redshifts of large numbers of galaxies. Obtaining spectroscopic redshifts for over $10^{8}$ galaxies is prohibitively timeconsuming, so we will rely on photometric redshifts estimated from the broadband optical imaging data. We have developed a photometric redshift estimation algorithm using boosted decision trees, which we have demonstrated outperforms the standard techniques used by earlier surveys such as the Sloan Digital Sky Survey. These improved photometric redshifts are now a standard part of DES simulations.

\section{NIR Lab}

The UM near-infrared (NIR) laboratory was begun nearly ten years ago to support the SNAP experiment, which later evolved into the Joint Dark Energy Mission (JDEM). This experiment planned to use a focal plane consisting in part of $\mathrm{HgCdTe}$ near-infrared detectors. During FY 2009-2012 the UM NIR addressed key technical issues related to the JDEM NIR detector program with a focus on precision photometry. R\&D activities concentrated on four areas: detector 'reciprocity failure', first reported by the HST-ACS team has been extensively investigated at Michigan and has revealed new inside in this phenomena and shown that this is an even larger problem as originally thought; a point projection system capable to measure the detailed pixel response for any potential JDEM NIR detector; automation of measurement of absolute detector quantum, conversion gain, dark current, and read; extension of measurements devices with cut-offs up to 2.1 micron.

\section{Reciprocity failure}

Count-rate-dependent nonlinearity, or reciprocity failure, is an example of nonlinearity in detector response that can limit photometric precision in astronomical observations. This effect was first reported for the $2.5 \mu \mathrm{m}$ cutoff Near Infrared Camera and Multi-Object Spectrometer (NICMOS) detectors and subsequently also verified in JDEM detectors (at our lab) and Wide Field Camera-3 detectors. Our UM group designed and built a dedicated reciprocity failure test system allowing us to perform precision linearity measurements on a series of detectors that were produced for the JDEM program. We 
were the first to point out the temperature dependence of this effect and the possibility of mitigation through cooling. Our detailed measurements show large variation in reciprocity failure between different detector runs and strong spatial non-uniformity in the reciprocity failure within individual detectors, indicating that the effect may be related to the material growth process. In particular the strong spatial non-uniformity puts in question the simple correction for this effect applied to the NICMOS detectors. Although the JDEM activities were terminated, our results are of great importance for any future photometric measurements with NIR detectors.

\section{Spots-O-Matic development}

The Spots-O-Matic is a multi spot projector designed to measure the internal response of every pixel in a $\mathrm{HgCdTe}$ detector. Effectively, we can measure the spatial variations in quantum efficiency on sub-pixel basis. This way even a star with a PSF that is small compared to the pixel size can have its flux accurately determined even if some areas of that pixel have lower sensitivity than others. In addition, knowledge of the sub-pixel structure is essential in performing weak lensing analysis with an undersampled imager since any variation in the pixel response could be confused with the PSF shape.

The Spots-o-Matic is the follow-up to a single spot projection system that was developed and successfully demonstrated in our lab. With the Spots-o-Matic we can focus and align a mask with 160,000 spots onto a detector. By rastering the spot mask and recording how the sensitivity of each pixel changes with position, a complete pixel-level sensitivity map of the detector is obtained.

To demonstrate the capability of the system, measurements were performed on a JDEM $\mathrm{HgCdTe}$ detector. This revealed a complex sub-pixel structure. Previous measurements made at Michigan using the single spot projection system showed that such structures could exist. In our latest measurement we observed a surprising effect: this structure appears to depend strongly on the total integrated charge within a pixel. To our knowledge such behavior has not been observed previously. If this behavior is common in $\mathrm{HgCdTe}$ detectors it could significantly impact the calibration needs of such devices.

In addition to characterizing sub-pixel structure, the Spots-O-Matic is capable to measure pixel size variations as well as other 2D structures that may bias shape determination in weak lensing applications (Pixel boundary shapes, scratches, photolithography mask beating).

\section{Absolute Quantum Efficiency}

Over the past year we have made significant improvements to our QE measurement setup mostly stemming from efforts to automate the procedure. As part of these efforts we have tightly integrated monochrometer and filter control as well as reference photodiode readout into the data acquisition scripts. This provides better repeatability and reduces the probability of operator errors. QE measurement used to be a rather time consuming process as diode calibration readings and filter band changes had to be done by hand. With this improved QE test set-up we were able to produce routine QE measurements on a short time scale with reduced manpower. The QE set-up can also be used for an 
automated conversion gain measurement. This allows us to efficiently and repeatedly characterize devices.

The improved QE setup has also given us an unprecedented ability to control various systematic effects. Real-time self-calibration during image acquisition allows us to control the total integrated signal to high precision so that we can eliminate, or alternatively study, the effects of charge integration nonlinearity. In addition, the uniform illumination with little thermal background and automatic neutral density filter selection has enabled reciprocity failure measurements to be performed at all wavelengths. Finally, using the QE setup we have also been able to determine that calibrated reference photodiodes often used for QE measurements can exhibit flux and wavelength dependent non-linearity when cooled to cryogenic temperatures.

\section{Measurements above 1.7 microns}

The proposed Reference Mission developed by the Interim Science Working Group has coverage in instrument wavelength from about 0.9 to 2 micron for the near infrared imager, allowing deeper redshift coverage for the BAO technique. While typical 1.7 micron detectors can be operated at $140 \mathrm{~K}, 2.1$ micron detectors need to be cooled to below $120 \mathrm{~K}$ to reach comparable dark current levels. This temperature can easily be accommodated in all our measurement set-ups, but for the measurement of detector QE we rely on calibrated $\mathrm{Si}$ and InGaAs photodiodes that were calibrated at a single temperature. Cold calibrated NIR photodiodes are not commercially available and thus a calibration transfer needed to be done. This required a dedicated set-up with a monochromator and two precision controlled temperature stages inside a dewar, with one stage at $300 \mathrm{~K}$ and the second, adjacent stage, at the target temperature. At $120 \mathrm{~K}$ this is a non-trivial measurement and required attention to every detail, as we want to preserve the quality of the calibration. Reference transfers were made from both warm $(295 \mathrm{~K})$ and $140 \mathrm{~K}$ to 140,120 and $100 \mathrm{~K}$ diodes. The newly calibrated reference diodes were used to characterize the 1.9 micron $\mathrm{H} 2 \mathrm{RG}-907$ device.

In order to reduce the thermal background during QE measurements and to allow a fully automated suite of measurements without having to open the dewar several times we designed and integrated an 'Universal Test Dewar' which allowed us to perform a complete characterization of a device.

\section{BigBOSS}

The study of baryon acoustic oscillations and the growth of structure with an all-sky galaxy redshift survey are powerful survey tools to investigate dark energy using groundbased facilities. The BigBOSS Stage IV dark energy experiment will study baryon acoustic oscillations and the growth of structure with an all-sky galaxy redshift survey. BigBOSS is planning to design and fabricate a 5,000-fiber spectrograph with associated focal plane and optical corrector system to be mounted on the Mayall telescope at Kitt Peak National Observatory (KPNO).

The University of Michigan is responsible for development of the BigBOSS calibration 
system, which will be used to initially calibrate the BigBOSS instrument, and Mayall telescope system, and will be used to periodically verify calibration of the system during science operations. We have designed and have begun to develop the necessary high precision spectrometric calibration system capable of providing a complete spectrophotometric calibration at the sub-pixel level for each spectrograph channel within an amount of time that will not negatively impact survey time. Our design uses a fast, high precision monochromator that can quickly and efficiently scan over the instrument's entire spectral range. It will have a spectral linewidth of less than 0.1Angstrom corresponding to a fraction of a pixel on the CCD. The proposed system delivers significant improvements over current calibration systems that typically use individual spectral lines from arc lamps and will improve relative wavelength calibration, absolute wavelength calibration, and most importantly point spread function mapping. This will allow understanding of the spectra to the theoretical noise limit.

\section{Ph.D. students:}

Jiangang Hao (graduated 2010, currently a research scientist at Fermilab)

Tomasz Biesiadzinski (expected graduation: 2014)

Adam Sypniewski (expected graduation: 2015)

\section{Publications:}

Precision Quantum Efficiency Measurements on HgCdTe Near Infrared Detectors, T. Biesiadzinski, W. Lorenzon, M. Schubnell, G. Tarlé, C. Weaverdyck, in preparation (2012)

Reciprocity Failure in HgCdTe Detectors: Measurements and Mitigation, T. Biesiadzinski, W. Lorenzon, R. Newman, M. Schubnell, G. Tarlé, C. Weaverdyck, PASP 123, 958 (2011) (2011).

Measurement of Reciprocity Failure in Near-Infrared Detectors, T. Biesiadzinski, W. Lorenzon, R. Newman, M. Schubnell, G. Tarlé, C. Weaverdyck, PASP 123, 179-186 (2011)

A method for the characterization of sub-pixel response of near-infrared detectors, Tomasz P. Biesiadzinski, Gregory Tarlé, Michael J. Howe, et al., Proc. SPIE 7742, 77421M (2010).

Investigating reciprocity failure in 1.7 -micron cut-off $\mathrm{HgCdTe}$ detectors, M. Schubnell, T. Biesiadzinski, W. Lorenzon, et al., Proc. SPIE, 7742, 77421N (2010).

ArborZ: Photometric Redshifts Using Boosted Decision Trees, D. Gerdes, A. Sypniewski, T. McKay, J. Hao, M. Weis, R. Wechsler, M. Busha, Ap. J. 715, 823 (2010).

Precision Measurements of the Cluster Red Sequence using an Error Corrected Gaussian Mixture Model, J. Hao et al., Ap. J. 702745 (2009). 
A GMBCG Galaxy Cluster Catalog of 55,424 Rich Clusters from SDSS DR7, J. Hao et al., Ap. J. Sup. 191, 254 (2010)

Reducing Zero-point Systematics in Dark Energy Supernova Experiments, L. Faccioli et al., AstroPart. Phys. 34, 847 (2011).

PHAT: PHoto-z Accuracy Testing, H. Hildebrandt et al., Astronomy \& Astrophysics 523, A31 (2010).

Photometric Redshifts in Non-Representative Galaxy Samples, A. J. Sypniewski and D. Gerdes, in preparation (2012).

Finding Fossil Groups: Optical Identification and X-Ray Confirmation, E. Miller et al., Ap. J. 747, 94 (2012).

Robust Optical Richness Estimation with Reduced Scatter, E. Rykoff et al., Ap. J. 746, 178 (2011).

Intrinsic Alignment of Cluster Galaxies: the Redshift Evolution, J. Hao et al., Ap. J. 740, 39 (2011).

Alignment of Brightest Cluster Galaxies with their Host Clusters, M. NiedersteOstholt el at., MNRAS 405, 2023 (2010).

Cosmological Constraints from the SDSS MaxBCG Catalog, E. Rozo et al., Ap. J. 708, 645 (2010).

Impact of Systematics on SZ-Optical Scaling Relations, T. Biesiadzinski et al, arXiv:1201.1282 (2012).

PreCam, A Precursor Observational Campaign for the Dark Energy Survey, arXiv:1208.0865 (2012).

\section{Talks:}

M. Schubnell, "Precision Quantum Efficiency Measurements on 1.7 Micron NIR Devices for JDEM/SNAP", Detectors for Astronomy workshop, ESO Garching, Germany, October 2009

M. Schubnell, Investigating Reciprocity failure in Near Infrared Detectors", SPIE Conference, San Diego, June 2010.

D. Gerdes, "The Dark Energy Survey," Shanghai Particle Astrophysics and Cosmology Conference, Shanghai, China, June 2011. 
Other:

G. Tarlé: member of Interim Science Working Group (ISWG); chair of calibration group. 


\section{High Energy Theory (Tasks G and T)}

The theory group is active in all areas from astrophysics and cosmology to the Standard Model, supersymmetry and string theory. The DoE supported faculty (FY09-12) are R. Akhoury, K. Freese, G. Kane, F. Larsen, J. Liu, L. Pando Zayas, A. Pierce, and J. Wells. The group has mentored numerous postdocs and graduate students over the last funding period.

\section{Research highlights}

The group's activities may be classified into four major categories - particle phenomenology, particle cosmology, string phenomenology and string theory/quantum gravity.

\subsection{Particle phenomenology}

- Kane, with post-doc Feldman, student Lu and B. Nelson (Northeastern), examined the implication for hints of Dark Matter for colliders. They have argued that the PAMELA data may point to a relatively light gluino, which would have important implications for the LHC.

- Kane, with former student Shao, visitor Petrov and Liantao Wang, proposed a new method to measure the spin of gluino candidates at the LHC that needed far less luminosity than other approaches, and was the leading method to test whether a discovery was indeed supersymmetry.

- $\quad$ Pierce and Wells, with student Jung, examined a tentative observation of a forwardbackward asymmetry in top quark production at the Tevatron. The standard model prediction for this quantity is small, so if the central value of the measurement persists, it would be a sign of new physics. This work explored one class of explanations and some attendant signals.

- With postdoc Feldman, Freese studied a predictive region of minimal supergravity and showed that DM and gluino masses can be inferred from kinematic edges in leptonic channels and peak values in effective mass distributions, as is being tested currently at the LHC.

\subsection{Particle cosmology}

- Freese and collaborators (including MCTP visitors Doug Spolyar and Paolo Gondolo) have proposed that the first stars (formed when the universe was 200 million years old) were powered by dark matter annihilation, rather than fusion. These Dark Stars, which would be a new phase of stellar evolution, could be detectable over the next decade, and much could be learned about dark matter if this is confirmed.

- Freese, Kane, Pierce and Zurek have all studied implications of recent hints of Dark Matter signals from both direct and indirect detection experiments. Pierce and Zurek have also been active in constructing new models of Dark Matter. Kane has argued that winolike dark matter is a strong candidate phenomenologically, and is also well motivated 
theoretically. Kane and Zurek have proposed different testable methods for baryogenesis and simultaneously relating the baryon number to the dark matter relic density.

- Akhoury with collaborators Saotome and Garfinkle studied the problem of gravitational collapse in theories of dynamical dark energy. They found an unusual feature of these models that the collapse proceeds through an intermediate stage of a black hole with two separate horizons, the usual light horizon and a second sonic horizon. In a separate work, Akhoury with another student Gauthier, developed a program to reconstruct the action for inflation with nonstandard kinetic energy for scalar fields using CMB data.

- Wells and collaborators have developed supersymmetric theories that satisfy big bang nucleosynthesis and dark matter abundance constraints while having a large enough reheat temperature to enable baryogenesis through the mechanism of thermal leptogenesis.

\subsection{String phenomenology}

- Kane and collaborators constructed a string based model with M-theory compactified on a manifold of $\mathrm{G}_{2}$ holonomy with moduli stabilized and supersymmetry broken, in which the TeV scale is derived rather than being input. They have calculated LHC and dark matter predictions, and shown there is no moduli or gravitino problem. They have argued that compactified string theories generically imply the universe has a non-thermal cosmological history. They have shown that there is a generic relation between moduli masses and the gravitino mass in all compactified string theories. This then requires scalars, including squarks, to have masses of order $30 \mathrm{TeV}$ or more, a firm LHC prediction, which has an associated prediction that the Higgs sector behaves like a single doublet with a calculable mass.

- Larsen, with student O’Connell and MCTP visitor Robbins, developed the moduli stabilization mechanism of type IIB vacua with ISD fluxes, finding an elegant expression for the stabilized moduli in terms of a single generating function.

- Akhoury and student Gauthier have shown that decoupling of heavy particle effects in theories with extra dimensions is not automatic but depends crucially on the type of compactification.

\subsection{String theory/quantum gravity}

- Liu, with Cremonini, Hanaki, and then student Szepietowski, studied higher derivative corrections to the shear viscosity to entropy density ratio $\eta / s$, using an off-shell formulation of 5D supergravity.

- Larsen, with Castro and (at the time MCTP visitor and now postdoc) Keeler, extended the CFT description of extreme Kerr black holes to excitations above the ground state. This is an important generalization because it allows the description of many physical processes that are frozen out in the extremal limit. 
- Larsen, with Balasubramanian, J. Simon and Etxebarria, identified a realization of the Luttinger liquid in the gravitational representation afforded by the AdS/CFT correspondence.

- Pando Zayas and collaborators, using the AdS/CFT correspondence, have launched the study of the far-from-equlibrium dynamics of thermalization in field theory via gravitational collapse in string theory. Pando Zayas and postdoc Reichmann constructed gravity duals generalizing unconventional $\mathrm{p}_{\mathrm{x}}$ and $\mathrm{p}_{\mathrm{x}}+\mathrm{i} \mathrm{p}_{\mathrm{y}}$ superconductors.

- Pando Zayas, with student Faraggi, found a universal one-loop description of supersymmetric Wilson loops using holography. Pando Zayas, with Caceres and Nunes, constructed a supergravity black hole that interpolates between the two most important gravity duals of confining theories at finite temperature.

- Akhoury, with collaborators Saotome and Sterman, has provided a systematic analysis of the nature of infrared divergences in perturbative quantum gravity amplitudes to all orders in perturbation theory. This has applications to high energy scattering in quantum gravity and to the relation of gauge theory amplitudes to those of perturbative quantum gravity.

\section{Postdocs}

Postdocs are an integral part of the theory group, and the group has been fortunate in attracting a large number of outstanding postdocs. Note, however, that the group has been able to leverage DoE funding with MCTP and University resources to enhance the impact of postdoc support. The following postdocs received at least partial DoE support during the last funding period:

- A. Ashoorion (07-09, then to Uppsala). Supported primarily by an outside fellowship.

- T. Flacke (07-09, then to Wurzburg). Some support from start-up funds.

- H. Lin (07-09, then to Santiago de Compostela).

- A. Menon (07-09, then to Illinois Institute of Technology).

- D. Reichmann (08-11). UM funds one year.

- D. Feldman (09-12). Some start-up funds.

- K. Kadota (09-11 then to faculty at Nagoya). Partially supported by UM funds.

- M. Park (09-11, then to U. Mass. at Amherst). Start-up funds one year.

- B. Wecht (10-11, then postdoc at Harvard and faculty at Queen Mary, London). Mostly start-up funds.

- $\quad$ H. Yu (10-). Mostly start-up funds.

- C. Keeler (11-).

- $\quad$ S. Tulin (11-). Mostly start-up funds.

There were five additional postdocs (S. Cremonini, G. Van Anders, S. Watson, I.-W. Kim and M. Gresham) associated with the group during at least part of the previous funding cycle. However, they were not directly supported by the DoE grant.

\section{Graduate students}


The Michigan theory group has long been training graduate students to relate theory and experimental phenomena. In formal theory, the group trains students to have a broad background that allows them to pursue different directions in the field. Graduate students are typically only partially supported by the DoE grant, with the remaining support obtained from fellowships and/or teaching. All students that received DoE support were active in research, and were actively involved in publications.

The following graduate students received partial DoE support during the last funding cycle:

- $\quad$ Stephen Amsel (Freese)

- Ibrahima Bah (Pando Zayas). PhD 2012, postdoc at USC/Saclay this fall.

- Alejandra Castro (Larsen). PhD 2009, postdoc at McGill and Harvard this fall.

- Tim Cohen (Pierce). PhD 2011, postdoc at SLAC.

- Jason Evans (Wells). PhD 2009, postdoc at IPMU.

- Alberto Faraggi (Pando Zayas). PhD 2012, postdoc at Universidade de Sao Paulo, Instituto de Fisica this fall.

- Christopher Gauthier (Akhoury). PhD 2010, postdoc at Laboratoire APC, Paris 7.

- Phill Grajeck (Kane). PhD 2009, postdoc at KEK.

- Sandeepan Gupta (Wells). PhD 2012.

- Kentaro Hanaki (Larsen). PhD 2012.

- Cosmin Ilie (Freese). PhD 2011.

- $\quad$ Sunghoon Jung (Wells).PhD 2011, postdoc at KAIST.

- John Kearney (Pierce).

- Eric Kuflik (Kane). PhD 2011, postdoc at Tel Aviv.

- Ran Lu (Kane).

- Ross O’Connell (Larsen). PhD 2010, postdoc at Carnegie Mellon.

- Daniel Phalen (Pierce). PhD 2010, postdoc at UC Davis.

- Ryo Saotome (Akhoury).

- Jing Shao (Kane). PhD 2009, postdoc at Syracuse.

- Phillip Szepietowski (Liu). PhD 2011, postdoc at Virginia.

- Bob Zheng (Kane).

- Zhichen Zhao (Liu). PhD 2012.

\section{The Michigan Center for Theoretical Physics}

The major activities of the MCTP are centered on workshops, conferences and visitors. Many activities are supported by a combination of MCTP and DoE funds.

\subsection{Doe supported MCTP workshops}

- Strings and gauge theories (September 15-26, 2008)

- $\quad$ LHC and dark matter 2009 (January 6-10, 2009)

- Great Lakes Strings Conference 2009 (April 3-5, 2009)

- Shining light on black holes (September 21-25, 2009)

- Micro dark matter workshop (January 28-30, 2010)

- Effective field theory in cosmology (March 11-13, 2010) 
- Non-thermal cosmological histories October 18-21, 2010)

- Amplitudes 2011 (November 10-13, 2011)

- Higgs workshop (April 16-20, 2012)

\subsection{Visitors}

- B. Acharya (long term visitor)

- C. Bambi

- A. Blechman

- K. Bobkov

- J. Collins

- C. Deffayet

- A. Dolgov (long term visitor)

- N. Halmagyi

- P. Kumar

- R. Leigh

- H. Liu

- R. Minasian

- B. Nelson

- H. de Oliveira

- M. Perry

- V. Rodgers

- P. Sandick

- O. Saremi

- D. Skinner

- D. Spolyar

- C. Terrero

- B. Thomas

- A. Vikman

\subsection{Young high energy theorists visitor program}

- L. Anguelova

- P. Basu

- T. Battefeld

- M. Buckley

- E. Cacares

- L. Carpenter

- C. Cheung

- S. Cremonini

- B. Czech

- T. Dumitrescu

- A. Frey

- J. Gainer 
- A. Gnecchi

- D. Green

- C.-M. Ho

- D. Hofman

- L.-Y. Hung

- J. Jottar

- D. Krohn

- T. Levi

- M. Lisanti

- S. Matsuura

- D. Robbins

- M. Roberts

- V. Rodgers

- J. Ruderman

- H.-H. Shieh

- G. Villadoro

- I. Yavin

\section{Publications}

The following publications were produced in the last funding period (FY09-12).

\subsection{Ratindranath Akhoury}

1. R. Akhoury, S. Mukohyama and R. Saotome, No Classicalization Beyond Spherical Symmetry, arXiv:1109.3820 [hep-th].

2. R. Akhoury, R. Saotome and G. Sterman, Collinear and soft divergences in perturbative quantum gravity, arXiv:1109.0270 [hep-th].

3. R. Akhoury, D. Garfinkle, R. Saotome and A. Vikman, Non-Stationary Dark Energy Around a Black Hole, Phys. Rev. D 83, 084034 (2011).

4. R. Akhoury, D. Garfinkle and R. Saotome, Gravitational collapse of k-essence, JHEP 1104, 096 (2011).

5. R. Saotome, R. Akhoury and D. Garfinkle, Examining gravitational collapse with test scalar fields, Class. Quant. Grav. 27, 165019 (2010).

6. R. Akhoury and A. D. Dolgov, On the Possibility of Super-luminal Propagation in a Gravitational Background, arXiv:1003.6110 [hep-th].

7. C. S. Gauthier, R. Saotome and R. Akhoury, Interaction of Neutrinos with a Cosmological Kessence Scalar, JHEP 1007, 062 (2010).

8. R. Akhoury, C. S. Gauthier and A. Vikman, Stationary Configurations Imply Shift Symmetry: No Bondi Accretion for Quintessence/K-Essence, JHEP 0903, 082 (2009).

\subsection{Katherine Freese}


1. K. Freese and C. Savage, Dark Matter collisions with the Human Body, arXiv:1204.1339 [astro-ph.CO].

2. B. W. Lynn, G. D. Starkman, K. Freese and D. I. Podolsky, A Goldstone 'Miracle': The Absense of a Higgs Fine Tuning Problem in the Spontaneously Broken O(4) Linear Sigma Model, arXiv:1112.2150 [hep-ph].

3. C. Ilie, K. Freese, M. Valluri, I. T. Iliev and P. Shapiro, Observing Dark Stars with JWST, arXiv:1110.6202 [astro-ph.CO].

4. A. Natarajan, C. Savage and K. Freese, Probing dark matter streams with CoGeNT, Phys. Rev. D 84, 103005 (2011) [arXiv:1109.0014 [astro-ph.CO]].

5. P. Sandick, J. Diemand, K. Freese and D. Spolyar, Gamma-Ray Constraints on the First Stars from Annihilation of Light WIMPs, Phys. Rev. D 85, 083519 (2012) [arXiv:1108.3820 [astro-ph.CO]].

6. S. Amsel, K. Freese and P. Sandick, Probing EWSB Naturalness in Unified SUSY Models with Dark Matter, JHEP 1111, 110 (2011) [arXiv:1108.0448 [hep-ph]].

7. D. Feldman, K. Freese, P. Nath, B. D. Nelson and G. Peim, Predictive Signatures of Supersymmetry: Measuring the Dark Matter Mass and Gluino Mass with Early LHC data, Phys. Rev. D 84, 015007 (2011) [arXiv:1102.2548 [hep-ph]].

8. P. Sandick, J. Diemand, K. Freese and D. Spolyar, Signatures of Dark Star Remnants in the Galactic Halo, PoS IDM2011, 086 (2011) [arXiv:1012.0068 [astro-ph.CO]].

9. P. Sandick, J. Diemand, K. Freese and D. Spolyar, Black Holes in our Galactic Halo: Compatibility with FGST and PAMELA Data and Constraints on the First Stars, JCAP 1101, 018 (2011) [arXiv:1008.3552 [astro-ph.CO]].

10. C. Ilie, K. Freese and D. Spolyar, Dark Stars and Boosted Dark Matter Annihilation Rates, New J. Phys. 13, 053050 (2011) [arXiv:1008.0348 [astro-ph.CO]].

11. K. Freese, E. Ruiz, M. Valluri, C. Ilie, D. Spolyar and P. Bodenheimer, Supermassive Dark Stars: Detectable in JWST and HST, AIP Conf. Proc. 1294, 45 (2010) [arXiv:1006.5246 [astro-ph.CO]].

12. C. Savage, G. Gelmini, P. Gondolo and K. Freese, XENON10/100 dark matter constraints in comparison with CoGeNT and DAMA: examining the Leff dependence, Phys. Rev. D 83, 055002 (2011) [arXiv:1006.0972 [astro-ph.CO]].

13. K. Kadota, K. Freese and P. Gondolo, Positrons in Cosmic Rays from Dark Matter Annihilations for Uplifted Higgs Regions in MSSM, Phys. Rev. D 81, 115006 (2010) [arXiv:1003.4442 [hep-ph]].

14. K. Freese, C. Ilie, D. Spolyar, M. Valluri and P. Bodenheimer, Supermassive Dark Stars: Detectable in JWST, Astrophys. J. 716, 1397 (2010) [arXiv:1002.2233 [astro-ph.CO]].

15. P. Sandick, D. Spolyar, M. R. Buckley, K. Freese and D. Hooper, The Sensitivity of the IceCube Neutrino Detector to Dark Matter Annihilating in Dwarf Galaxies, Phys. Rev. D 81, 083506 (2010) [arXiv:0912.0513 [astro-ph.CO]].

16. S. K. Mandal, M. R. Buckley, K. Freese, D. Spolyar and H. Murayama, Cascade Events at IceCube+DeepCore as a Definitive Constraint on the Dark Matter Interpretation of the 
PAMELA and Fermi Anomalies, Phys. Rev. D 81, 043508 (2010) [arXiv:0911.5188 [hep$\mathrm{ph}]]$.

17. C. Bambi, K. Freese, T. Harada, R. Takahashi and N. Yoshida, Accretion process onto superspinning objects, Phys. Rev. D 80, 104023 (2009) [arXiv:0910.1634 [gr-qc]].

18. C. Bambi, K. Freese and R. Takahashi, Is the Carter-Israel conjecture correct?, arXiv:0908.3238 [astro-ph.HE].

19. C. Ilie, T. Biswas and K. Freese, Are we seeing the beginnings of inflation?, Phys. Rev. D 80, 103521 (2009) [arXiv:0908.0991 [astro-ph.CO]].

20. T. Flacke, A. Menon, D. Hooper and K. Freese, Kaluza-Klein Dark Matter and Neutrinos from Annihilation in the Sun, arXiv:0908.0899 [hep-ph].

21. M. R. Buckley, K. Freese, D. Hooper, D. Spolyar and H. Murayama, High-Energy Neutrino Signatures of Dark Matter Decaying into Leptons, Phys. Rev. D 81, 016006 (2010) [arXiv:0907.2385 [astro-ph.HE]].

22. D. Spolyar, M. R. Buckley, K. Freese, D. Hooper and H. Murayama, High Energy Neutrinos as a Test of Leptophilic Dark Matter, arXiv:0905.4764 [astro-ph.CO].

23. D.-C. Dai, K. Freese and D. Stojkovic, Constraints on dark matter particles charged under a hidden gauge group from priordial black holes, JCAP 0906, 023 (2009) [arXiv:0904.3331 [hep-ph]].

24. D. Spolyar, P. Bodenheimer, K. Freese and P. Gondolo, Dark Stars: A new look at the First Stars in the Universe, Astrophys. J. 705, 1031 (2009) [arXiv:0903.3070 [astro-ph.CO]].

25. K. Freese, D. Spolyar, P. Bodenheimer and P. Gondolo, Dark Stars: A New Study of the First Stars in the Universe, New J. Phys. 11, 105014 (2009) [arXiv:0903.0101 [astro-ph.CO]].

26. P. Gondolo, K. Freese, D. Spolyar, A. Aguirre, P. Bodenheimer, J. A. Sellwood and N. Yoshida, Dark Stars: Begynnelsen, PoS IDM2008, 074 (2008) [arXiv:0901.4578 [astroph.CO]].

27. D. Spolyar, K. Freese, P. Gondolo, A. Aguirre, P. Bodenheimer, J. A. Sellwood and N. Yoshida, Dark Stars: Dod och Ateruppstandelse, PoS IDM2008, 077 (2008) [arXiv:0901.4574 [astro-ph.CO]].

28. C. Savage, K. Freese, P. Gondolo and D. Spolyar, Compatibility of DAMA/LIBRA dark matter detection with other searches in light of new Galactic rotation velocity measurements, JCAP 0909, 036 (2009) [arXiv:0901.2713 [astro-ph]].

29. K. Freese, P. Bodenheimer, P. Gondolo and D. Spolyar, Dark Stars: The First Stars in the Universe may be powered by Dark Matter Heating, AIP Conf. Proc. 1166, 33 (2009) [arXiv:0812.4844 [astro-ph]].

30. K. Freese, Review of Observational Evidence for Dark Matter in the Universe and in upcoming searches for Dark Stars, arXiv:0812.4005 [astro-ph].

31. C. Bambi and K. Freese, Apparent shape of super-spinning black holes, Phys. Rev. D 79, 043002 (2009) [arXiv:0812.1328 [astro-ph]]. 
32. C. Bambi, D. Spolyar, A. D. Dolgov, K. Freese and M. Volonteri, Implications of primordial black holes on the first stars and the origin of the super-massive black holes, Mon. Not. Roy. Astron. Soc. 399, 1347 (2009) [arXiv:0812.0585 [astro-ph]].

33. A. Ashoorioon and K. Freese, Gravity Waves from Chain Inflation, arXiv:0811.2401 [hepth].

\subsection{Gordon Kane}

1. Compactified Strings Theories - Generic Predictions for Particle Physics, B. S. Acharya, G. Kane, P. Kumar, Int. J. Mod. Phys. A 27 (2012) 1230012 [arXiv:1204.2795 [hep-ph]].

2. Discovering Gluino Events at LHC-8 via Disappearing Chargino Tracks, G. Kane, R. Lu, B. Zheng, arXiv:1202.4448 [hep-ph].

3. Higgs Mass Prediction for Realistic String/M-Theory Vacua, G. Kane, P. Kumar, R. Lu, B. Zheng, Phys. Rev. D 85 (2012) 075026 [arXiv:1112.1059 [hep-ph]].

4. The Baryon-Dark Matter Ratio via Moduli Decay After Affleck-Dine Baryogenesis, by Gordon Kane, Jing Shao, Scott Watson, Hai-Bo Yu, arXiv:1108.5178.

5. Flavour issues for string-motivated heavy scalar spectra with a low gluino mass: the G2MSSM case, by Kenji Kadota, Gordon Kane, Joern Kersten, Liliana Velasco-Sevilla, arXiv:1107.3105.

6. Extracting the Wavefunction of the LSP at the LHC, by Gordon Kane, Eric Kuflik, Brent Nelson, Phys. Lett. B 703 (2011) 151 [arXiv:1105.3742].

7. A New (String motivated) approach to the Little Hierarchy Problem, by Daniel Feldman, Gordon Kane, Eric Kuflik, Ran Lu, Phys. Lett. B 704 (2011) 56 [arXiv:1105.3765].

8. Top Channel for Early SUSY discovery at the LHC , by Gordon Kane, Eric Kuflilk, Ran Lu, and Liantao Wang, arXiv:1101.1963.

9. The $\mu$ parameter in M-theory: Theory and Phenomenology, by Bobby Acharya, Gordon Kane, and Erik Kuflik, arXiv:1102.0556, JHEP 1105 (2011) 033.

10. String Theories with Moduli Stabilization Imply Non-Thermal Cosmological History, and Particular Dark Matter, by Bobby Samir Acharya, Gordon Kane, Eric Kuflik, arXiv:1006.3272.

11. Dark Matter as a Guide Toward a Light Gluino at the LHC, by Daniel Feldman, Gordon Kane, Ran Lu, Brent D. Nelson, Phys. Lett. B 687, 363 (2010) [arXiv:1002.2430].

12. The Hunt for New Physics at the Large Hadron Collider, by P. Nath, et.al., [arXiv:1001.2693] (Jan 2010).

13. A Non-thermal WIMP Miracle, by Bobby Samir Acharya, Gordon Kane, Scott Watson, Piyush Kumar, Phys. Rev. D 80, 083529 (2009) [arXiv:0908.2430].

14. PAMELA Satellite Data as a Signal of Non-Thermal Wino LSP Dark Matter, by Gordon Kane, Ran Lu, Scott Watson. Phys. Lett. B 681, 151 (2009) [arXiv:0906.4765].

15. CP-violating Phases in M-theory and Implications for EDMs, by Gordon Kane, Piyush Kumar, Jing Shao, Phys. Rev. D 82, 055005 (2010) [arXiv:0905.2986]. 
16. The Footprint of F-theory at the LHC, by Jonathan J. Heckman, Gordon L. Kane, Jing Shao, Cumrun Vafa, JHEP 0910, 039 (2009) [arXiv:0903.3609].

17. Identifying Multi-Top Events from Gluino Decay at the LHC, by Bobby Samir Acharya, Phill Grajek, Gordon L. Kane, Eric Kuflik, Kerim Suruliz, Lain-Tao Wang, [arXiv:0901.3367] MCTP-09-03 (Jan 2009).

18. Studying Gaugino Mass unification at the LHC, by Baris Altunkaynak, Phillip Grajek, Michael Holmes, Gordon Kane, Brent D. Nelson, JHEP 0904, 114 (2009) [arXiv:0901.11458].

19. Is the PAMELA Positron Excess Winos? by Phill Grajek, Gordon Kane, Dan Phalen, Aaron Pierce, Scott Watson, Phys. Rev. D 79, 043506 (2009) [arXiv:0812.4555].

20. The LHC: A “Why” Machine and a Supersymmetry Factory, by Gordon Kane. In Kane, Gordon (ed.) et al., Perspectives on LHC Physics 1-11.

\subsection{Finn Larsen}

1. E. Gimon, F. Larsen and J. Simon. Constituent Model of Extremal non-BPS Black Holes, JHEP 0907, 052 (2009) [arXiv:0903.0719 [hep-th]].

2. F. Larsen and R. O’Connell. Flux Attractors and Generating Functions, JHEP 0907, 049 (2009) [arXiv:0905.2130 [hep-th]].

3. A. Castro and F. Larsen. Near Extremal Kerr Entropy from AdS $S_{2}$ Quantum Gravity, JHEP 0912, 037 (2009) [arXiv:0908.1121 [hep-th]].

4. M. Cvetic and F. Larsen. Greybody Factors and Charges in Kerr/CFT, JHEP 0909, 088 (2009) [arXiv:0908.1136 [hep-th]].

5. F. Larsen, R. O’Connell and D. Robbins. Hypermoduli Stabilization, Flux Attractors and Generating Functions, JHEP 1006, 077 (2010) [arXiv:0912.4448 [hep-th]].

6. A. Castro, F. Larsen and C. Keeler, Three Dimensional Origin of AdS ${ }_{2}$ Quantum Gravity, JHEP 1007, 033 (2010) [arXiv:1004.0554 [hep-th]].

7. F. Larsen and G. v. Anders, Holographic Non-Fermi Liquids and the Luttinger Theorem, arXiv:1006.1846 [hep-th].

8. L. Anguelova, F. Larsen and R. O’Connell, Heterotic Flux Attractors, JHEP 1011, 010 (2010) [arXiv:1006.4981 [hep-th]].

9. V. Balasubramanian, I. Garcia-Etxebarria, F. Larsen and J. Simon, Helical Luttinger Liquids and Three-Dimensional Black Holes, Phys. Rev. D 84, 126012 (2011) [arXiv:1012.4363 [hep-th]].

10. M. Cvetic and F. Larsen, Conformal Symmetry for General Black Holes, arXiv:1106.3341 [hep-th].

11. M. Cvetic and F. Larsen, Conformal Symmetry for Black Holes in Four Dimensions, arXiv:1112.4846 [hep-th].

\subsection{James Liu}

1. N. Halmagyi, J. T. Liu and P. Szepietowski, On $N=2$ Truncations of IIB on $T^{1,1}$, JHEP 1207, 
098 (2012) [arXiv:1111.6567 [hep-th]].

2. J. T. Liu and Z. Zhao, A holographic c-theorem for higher derivative gravity, arXiv:1108.5179 [hep-th].

3. J. T. Liu and P. Szepietowski, Supersymmetry of consistent massive truncations of IIB supergravity, arXiv:1103.0029 [hep-th].

4. J. T. Liu, W. Sabra and Z. Zhao, Holographic c-theorems and higher derivative gravity, Phys. Rev. D 85, 126004 (2012) [arXiv:1012.3382 [hep-th]].

5. J. T. Liu and R. Minasian, Computing 1/N ${ }^{2}$ corrections in AdS/CFT, arXiv:1010.6074 [hepth].

6. J. T. Liu, P. Szepietowski and Z. Zhao, Supersymmetric massive truncations of IIB supergravity on Sasaki-Einstein manifolds, Phys. Rev. D 82, 124022 (2010) [arXiv:1009.4210 [hep-th]].

7. J. T. Liu and C. N. Pope, Inconsistency of Breathing Mode Extensions of Maximal FiveDimensional Supergravity Embedding, JHEP 1206, 067 (2012) [arXiv:1005.4654 [hep-th]].

8. J. T. Liu, P. Szepietowski and Z. Zhao, Consistent massive truncations of IIB supergravity on Sasaki-Einstein manifolds, Phys. Rev. D 81, 124028 (2010) [arXiv:1003.5374 [hep-th]].

9. S. Cremonini, J. T. Liu and P. Szepietowski, Higher Derivative Corrections to R-charged Black Holes: Boundary Counterterms and the Mass-Charge Relation, JHEP 1003, 042 (2010) [arXiv:0910.5159 [hep-th]].

10. S. Cremonini, K. Hanaki, J. T. Liu and P. Szepietowski, Higher derivative effects on $\eta / s$ at finite chemical potential, Phys. Rev. D 80, 025002 (2009) [arXiv:0903.3244 [hep-th]].

11. S. Cremonini, K. Hanaki, J. T. Liu and P. Szepietowski, Black holes in five-dimensional gauged supergravity with higher derivatives, JHEP 0912, 045 (2009) [arXiv:0812.3572 [hepth]].

\subsection{Leopoldo Pando Zayas}

1. P. Basu, D. Das, A. Ghosh and L. A. Pando Zayas, Chaos around Holographic Regge Trajectories, JHEP 1205 (2012) 077 [arXiv:1201.5634 [hep-th]].

2. A. Faraggi, W. Mueck and L. A. Pando Zayas, One-loop Effective Action of the Holographic Antisymmetric Wilson Loop, Phys. Rev. D 85 (2012) 106015 [arXiv:1112.5028 [hep-th]].

3. D. Garfinkle, L. A. Pando Zayas and D. Reichmann, On Field Theory Thermalization from Gravitational Collapse, JHEP 1202 (2012) 119 [arXiv:1110.5823 [hep-th]].

4. L. A. Pando Zayas and D. Reichmann, A Holographic Chiral $p_{x}+i p_{y}$ Superconductor, Phys. Rev. D 85 (2012) 106012 [arXiv:1108.4022 [hep-th]].

5. D. Garfinkle and L.A. Pando Zayas, Rapid Thermalization in Field Theory from Gravitational Collapse, Phys. Rev. D 84 (2011) 066006 [arXiv:1106.2339 [hep-th]].

6. P. Basu and L. A. Pando Zayas, Analytic Non-integrability in String Theory, Phys. Rev. D 84 (2011) 046006 [arXiv:1105.2540 [hep-th]]. 
7. P. Basu and L. A. Pando Zayas, Chaos rules out integrability of strings in $A d S_{5} \times T^{1,1}$, Phys. Lett. B 700 (2011) 243 [arXiv:1103.4107 [hep-th]].

8. A. Faraggi and L. A. Pando Zayas, The Spectrum of Excitations of Holographic Wilson Loops, JHEP 1105 (2011) 018 [arXiv:1101.5145 [hep-th]].

9. E. Caceres, C. Nunez and L .A. Pando Zayas, Heating up the Baryonic Branch with Uduality: a unified picture of conifold black holes, JHEP 1103 (2011) 054 [arXiv:1101.4123 [hep-th]].

10. B. Burrington and L. A. Pando Zayas, Phase transitions in Wilson loop correlator from integrability in global AdS, Int. J. Mod. Phys. A 27 (2012) 1250001 [arXiv:1012.1525 [hepth]].

11. I. Bah, A. Faraggi, J. Jottar, R. Leigh and L. A. Pando Zayas, Fermions in $D=11$ Supergravity on squashed Sasaki-Einstein manifolds, JHEP 1102 (2011) 068 [arXiv:1008.1423 [hep-th]].

12. E. Caceres, M. N. Mahato, L.A. Pando Zayas and V. J.G. Rodgers, Toward NS5 Branes on the Resolved Cone over $Y^{p, q}$, Phys. Rev. D 83 (2011) 066008 [arXiv:1007.3719 [hep-th]].

13. L. A. Pando Zayas and C. A. Terrero-Escalante, Chaos in the Gauge / Gravity Correspondence, JHEP 1009 (2010) 094 [arXiv:1007.0277 [hep-th]].

14. J. Jottar, R. G. Leigh, and D. Minic and L. A. Pando Zayas, Aging and Holography, JHEP 1011 (2010) 034 [arXiv:1004.3752 [hep-th]].

15. C. Doran, L. A. Pando Zayas, V. Rodgers and K. Stiffler, Tensions and Luscher Terms for (2+1)-dimensional k-strings from Holographic Models, 0911 (2009) 064 [arXiv:0907.1331 [hep-th]].

\subsection{Aaron Pierce}

1. T. Cohen, D. E. Morrissey, A. Pierce, "Electroweak Baryogenesis and Higgs Signatures,” arXiv:1203.2924 [hep-ph].

2. J. Kearney, A. Pierce, "Neutrinos from Off-Shell Final States and the Indirect Detection of Dark Matter,” arXiv:1202.0284 [hep-ph].

3. T. Cohen, A. Pierce, "Electroweak Baryogenesis and Colored Scalars,” Phys. Rev. D 85, 033006 (2012) [arXiv:1110.0482 [hep-ph]].

4. T. Cohen, J. Kearney, A. Pierce, D. Tucker-Smith, “Singlet-Doublet Dark Matter," Phys. Rev. D 85, 075003 (2012) [arXiv:1109.2604 [hep-ph]].

5. S. Jung, A. Pierce, J. D. Wells, "Top asymmetry and the search for a light hadronic resonance in association with single top,” Phys. Rev. D 84, 091502 (2011) [arXiv:1108.1802 [hep-ph]].

6. S. Jung, A. Pierce, J. D. Wells, "Top quark asymmetry and dijet resonances," Phys. Rev. D 84, 055018 (2011) [arXiv:1104.3139 [hep-ph]].

7. S. Jung, A. Pierce, J. D. Wells, “Top quark asymmetry from a non-Abelian horizontal symmetry,” Phys. Rev. D 83, 114039 (2011). [arXiv:1103.4835 [hep-ph]]. 
8. M. A. Luty, D. J. Phalen, A. Pierce, "Natural $h \rightarrow 4 g$ in Supersymmetric Models and RHadrons at the LHC,” Phys. Rev. D 83, 075015 (2011). [arXiv:1012.1347 [hep-ph]].

9. T. Cohen, D. J. Phalen, A. Pierce, K. M. Zurek, “Asymmetric Dark Matter from a GeV Hidden Sector,” Phys. Rev. D 82, 056001 (2010). [arXiv:1005.1655 [hep-ph]].

10. S. Chang, J. Liu, A. Pierce, N. Weiner, I. Yavin, “CoGeNT Interpretations,” JCAP 1008, 018 (2010). [arXiv:1004.0697 [hep-ph]].

11. E. Kuflik, A. Pierce, K. M. Zurek, "Light Neutralinos with Large Scattering Cross Sections in the Minimal Supersymmetric Standard Model,” Phys. Rev. D 81, 111701 (2010). [arXiv:1003.0682 [hep-ph]].

12. T. Cohen, D. J. Phalen, A. Pierce, "On the Correlation Between the Spin-Independent and Spin-Dependent Direct Detection of Dark Matter,” Phys. Rev. D 81, 116001 (2010). [arXiv:1001.3408 [hep-ph]].

13. S. Chang, A. Pierce, N. Weiner, “Momentum Dependent Dark Matter Scattering,” JCAP 1001, 006 (2010). [arXiv:0908.3192 [hep-ph]].

14. T. Cohen, D. J. Phalen, A. Pierce, "Supersymmetric Baryogenesis from Exotic Quark Decays”, Phys. Rev. D 81, 035020 (2010) [arXiv:0908.2998 [hep-ph]].

15. S. Jung, H. Murayama, A. Pierce, J. D. Wells, “Top quark forward-backward asymmetry from new t-channel physics,” Phys. Rev. D 81, 015004 (2010). [arXiv:0907.4112 [hep-ph]].

16. A. Menon, R. Morris, A. Pierce, N. Weiner, "Capture and Indirect Detection of Inelastic Dark Matter,” Phys. Rev. D 82, 015011 (2010). [arXiv:0905.1847 [hep-ph]].

17. D. J. Phalen, A. Pierce, N. Weiner, “Cosmic Ray Positrons from Annihilations into a New, Heavy Lepton,” Phys. Rev. D 80, 063513 (2009). [arXiv:0901.3165 [hep-ph]].

18. P. Grajek, G. Kane, D. Phalen, A. Pierce, S. Watson, “Is the PAMELA Positron Excess Winos?,” Phys. Rev. D 79, 043506 (2009). [arXiv:0812.4555 [hep-ph]].

\subsection{James Wells}

1. R. S. Gupta and J. D. Wells, Higgs boson search significance deformations due to mixed-in scalars, Phys. Lett. B 710, 154 (2012) [arXiv:1110.0824 [hep-ph]].

2. S. Jung, A. Pierce and J. D. Wells, Top asymmetry and the search for a light hadronic resonance in association with single top, Phys. Rev. D 84, 091502 (2011) [arXiv:1108.1802 [hep-ph]].

3. S. Jung, A. Pierce and J. D. Wells, Top quark asymmetry and dijet resonances, Phys. Rev. D 84, 055018 (2011) [arXiv:1104.3139 [hep-ph]].

4. S. Jung, A. Pierce and J. D. Wells, Top quark asymmetry from a non-Abelian horizontal symmetry, Phys. Rev. D 83, 114039 (2011) [arXiv:1103.4835 [hep-ph]].

5. L. Covi, M. Olechowski, S. Pokorski, K. Turzynski and J. D. Wells, Supersymmetric mass spectra for gravitino dark matter with a high reheating temperature, JHEP 1101, 033 (2011) [arXiv:1009.3801 [hep-ph]]. 
6. S. Jung and J. D. Wells, Low-scale warped extra dimension and its predilection for multiple top quarks, JHEP 1011, 001 (2010) [arXiv:1008.0870 [hep-ph]].

7. R. S. Gupta and J. D. Wells, Next Generation Higgs Bosons: Theory, Constraints and Discovery Prospects at the Large Hadron Collider, Phys. Rev. D 81, 055012 (2010) [arXiv:0912.0267 [hep-ph]].

8. M. Olechowski, S. Pokorski, K. Turzynski and J. D. Wells, Reheating Temperature and Gauge Mediation Models of Supersymmetry Breaking, JHEP 0912, 026 (2009) [arXiv:0908.2502 [hep-ph]].

9. S. Jung, H. Murayama, A. Pierce and J. D. Wells, Top quark forward-backward asymmetry from new t-channel physics, Phys. Rev. D 81, 015004 (2010) [arXiv:0907.4112 [hep-ph]].

10. Y. Cui, T. Gherghetta and J. D. Wells, Emergent Electroweak Symmetry Breaking with Composite W, Z Bosons, JHEP 0911, 080 (2009) [arXiv:0907.0906 [hep-ph]].

11. S. Gopalakrishna, S. J. Lee and J. D. Wells, Dark matter and Higgs boson collider implications of fermions in an abelian-gauged hidden sector, Phys. Lett. B 680, 88 (2009) [arXiv:0904.2007 [hep-ph]].

12. I. ntoniadis, A. Boyarsky, S. Espahbodi, O. Ruchayskiy and J. D. Wells, Anomaly driven signatures of new invisible physics at the Large Hadron Collider, Nucl. Phys. B 824, 296 (2010) [arXiv:0901.0639 [hep-ph]].

13. J. L. Evans, D. E. Morrissey and J. D. Wells, Vacuum Stability with Tachyonic Boundary Higgs Masses in No-Scale Supersymmetry or Gaugino Mediation, Phys. Rev. D 80, 095011 (2009) [arXiv:0812.3874 [hep-ph]].

14. S. Jung and J. D. Wells, Comparison of electric dipole moments and the Large Hadron Collider for probing CP violation in triple boson vertices, Phys. Rev. D 80, 015009 (2009) [arXiv:0811.4140 [hep-ph]]. 


\title{
Task J: Probing the Nature of Dark Energy
}

\author{
PI: Dragan Huterer \\ Grant closeout report for period 2009-2011
}

In 2009-2011 Task J funded the PI Dragan Huterer and his postdoc Carlos Cunha. [Cunha is now Kavli Fellow at Stanford University, and the PI hired Hao-Yi (Heidi) Wu as the new postdoc, starting in 2011.] The principal area of PI's research has been dark energy phenomenology, as well as studies of non-Gaussianity as a probe of the early universe. The principal findings for the 2009-2011 period are:

- In a series of papers, Huterer (together with Michael Mortonson from Ohio State University and Wayne $\mathrm{Hu}$ from the University of Chicago) made predictions of general classes of dark energy models for the distance and growth functions as a function of cosmic time. This has been done for the current data, and also forecasted for future data. We made quantitative and nearly model-independent predictions for several classes of dark energy models (Lambda cold dark matter $(\Lambda \mathrm{CDM})$, quintessence, etc). We also studied the generalized figures of merit for dark energy in the context of the current and future data and found that, with future space-based data, the constraints on dark energy models will improve for approximately 6 independent dark energy parameters resulting in a reduction of the total allowed volume of dark energy parameter space by a factor of $\sim 100$. Finally, we have studied variations in the dark energy density at recent times (i.e. low redshift) would appear in cosmological observations.

- Mortonson, Hu and Huterer also clarified how to use the high-mass, high-redshift clusters of galaxies (the 'pink elephant' clusters) to test consistency with cosmological models. Previous analyses argued that the existence of these galaxy clusters formally rules out the cosmologicalconstant dominated universe at $2-4 \sigma$, since such massive, distant objects are not expected to exist in the currently favored $\Lambda \mathrm{CDM}$ model. After careful analysis, we found that all currently observed clusters are in fact concordant with $\Lambda$ CDM. We made available formulae that quantify the level of agreement between any new observations and theory as a function of survey parameters. These results have already been used by the South Pole Telescope team to interpret their new observations.

- Huterer and collaborators studied the effects of the dark energy speed of sound on cosmological observations, and the extent to which it can be measured. The PI also studied the effects of early dark energy (dark energy that is not negligible at earlier epochs in the history of the universe, say around recombination).

- Huterer and collaborators studied catastrophic redshift errors - large outliers between the true and photometrically inferred redshifts. Catastrophic photo-z errors lead to systematic biases in the dark energy parameters, and we investigated how accurately these pervasive errors need to be controlled in order not to affect the dark energy measurements. Very recently, the PI and his former OJI-funded postdoc Cunha quantified, for the first time, sample variance errors that the large-scale structure causes photometric calibration of surveys. This work quantifies how much spectroscopic follow-up (for surveys such as DES or LSST) is required in order that dark energy constraints not be biased.

- Huterer and collaborators set up a formalism to study the statistical isotropy of the universe using large-scale structure, and test this fundamental premise in cosmology more stringently than ever done before. This formalism uses multipole vectors, which we had previously introduced 
to cosmology and used to study alignments in cosmic microwave background (CMB) maps. In our new work, we tested the method on synthetic maps, and studied the accuracy of constraints with respect to sky coverage, galaxy number density, and resolution at which the tests are performed. In an ongoing work, we are applying the studies on various maps of large-scale structure (galaxies, clusters, gamma-ray bursts).

- Huterer and collaborators have uncovered a novel signature of primordial non-Gaussianity: strong scale dependence of the dark matter halo bias (Dalal, Doré, Huterer \& Shirokov, Phys. Rev. $D, \mathbf{7 7}, 123514,2008)$. This work pointed to a new and powerful way to test non-Gaussianity; using this effect, the constraints on non-Gaussianity from the large-scale structure (LSS) are about two orders of magnitude better than previously thought possible. Our work led to a revived interest in developing theory, simulations, and observations to search for signatures of classes of inflationary models using astrophysical observations of the LSS. The PI and collaborators also studied how well scale-dependent models of non-Gaussianity can be probed by a combination of the LSS and CMB. The PI was the principal organizer of the workshop on primordial non-Gaussianity at the University of Michigan in May 2011, which around 70 of the world's top experts on the subject attended.

- In 2009-2011 Huterer served as the Lead Guest Editor of a Special Issue of Advances in Astronomy (Hindawi Publishing Corporation). Together with the other Guest Editors Eiichiro Komatsu and Sarah Shandera, Huterer edited a volume on "Testing the Gaussianity and Statistical Isotropy of the Universe" (see http://www.hindawi.com/journals/aa/2010/si.gsiu.html). The volume includes a total of 15 articles on all aspects of this topic from leading experts in the field, and the hope is that these articles, which have already been cited more than 500 times, will serve as a standard hands-on reference for researchers in the field.

\section{Publications in 2009-2011:}

1. "The BigBOSS Experiment", D. Schlegel et al., arXiv:1106.1706

2. "Limits on Dark Radiation, Early Dark Energy, and Relativistic Degrees of Freedom", E. Calabrese, D. Huterer, E.V. Linder, A. Melchiorri, and L. Pagano, Phys. Rev. D, 83, 023011 (2011)

3. "Bias in low-multipole CMB reconstructions", C.J. Copi, D. Huterer , D.J. Schwarz, and G.D. Starkman, MNRAS, 418, 505 (2011)

4. "Robustness to systematics for future dark energy probes", M. March, R. Trotta, L. Amendola and D. Huterer, MNRAS, 415, 143 (2011)

5. "Simultaneous Falsification of LambdaCDM and Quintessence with Massive, Distant Clusters", M. Mortonson, W. Hu and D. Huterer, Phys. Rev. D, 83, 023015 (2011)

6. "Future CMB Constraints on Early, Cold, or Stressed Dark Energy", E. Calabrese, R. de Putter, D. Huterer, E.V. Linder and A. Melchiorri, Phys. Rev. D, 83, 023011 (2011)

7. "A generalized local ansatz and its effect on halo bias", S. Shandera, N. Dalal and D. Huterer, JCAP, 1103:017 (2011)

8. "Testing the statistical isotropy of large scale structure with multipole vectors ", C. Zunckel, D. Huterer and G.D. Starkman, Phys. Rev. D, 84, 043005 (2011)

9. "Scale-Dependent Non-Gaussianity as a Generalization of the Local Model", A. Becker, D. Huterer and K. Kadota, JCAP, 1101:006 (2011)

10. "Detectability of large-scale power suppression in the galaxy distribution", C. Gibelyou, D. Huterer and W. Fang, Phys. Rev. D, 82, 013009 (2010) 
11. "Large-scale anomalies in the CMB", C.J. Copi, D. Huterer, D.J. Schwarz, and G.D. Starkman, Adv. Astronomy, Vol. 2010, Article ID 847541 (2010)

12. "Missing Power vs low-l Alignments in the Cosmic Microwave Background: No Correlation in the Standard Cosmological Model", D. Sarkar, D. Huterer, C.J. Copi, G.D. Starkman and D.J. Schwarz, Astroparticle Phys., 34, 591 (2010)

13. "Figures of merit for present and future dark energy probes", M. Mortonson, D. Huterer and W. Hu, Phys. Rev. D, 82, 063004 (2010)

14. "Primordial non-Gaussianity from the covariance of galaxy cluster counts", C. Cunha, D. Huterer and O. Doré, Phys. Rev. D, 82, 023004 (2010)

15. "A General Study of The Influence of Catastrophic Photometric Redshift Errors on Cosmology with Cosmic Shear Tomography" A. Hearin, A. Zentner, Z. Ma, and D. Huterer, Astrophys. J., 720, 1351 (2010)

16. "Measuring the Speed of Dark: Detecting Dark Energy Perturbations", R. de Putter, D. Huterer and E. Linder, Phys. Rev. D, 82, 023004 (2010)

17. "Weak lensing, dark matter and dark energy", D. Huterer, Gen. Rel. Grav., 42, 2177 (2010)

18. "Testable dark energy predictions from current data", M. Mortonson, W. Hu and D. Huterer, Phys. Rev. D, 81, 063007 (2010)

19. "Disks in the sky: A reassessment of the WMAP 'cold spot' ", R. Zhang and D. Huterer, Astroparticle Phys., 33, 69 (2010)

20. "Hiding dark energy transitions at low redshift", M. Mortonson, W. Hu and D. Huterer, Phys. Rev. D, 79, $067301(2009)$

21. "Constraining Dark Energy with Clusters: Complementarity with Other Probes", C. Cunha, D. Huterer and J.A. Frieman, Phys. Rev. D, 80, 064532 (2009)

22. "Catastrophic photometric redshift errors: weak lensing survey requirements", G. Bernstein and D. Huterer, MNRAS, 401, 1399 (2010)

\section{Major talks and lectures, 2009-2011:}

- Invited speaker at the "Pre-Planckian Inflation 2011" workshop, University of Minnesota (October 2011)

- Lecturer at the cosmology summer schools at the Azores (August 2011)

- Invited participant at the "DEUS: current and future challenges of the Dark and Early Universes" workshop at the Dark Cosmology Centre in Copenhagen, Denmark (August 2011)

- Principal organizer of the "Cosmological Non-Gaussianity: Observations Confront Theory" Workshop at the University of Michigan (May 2011)

- Panelist at the Experiments on the Cosmic Frontier symposium at Fermilab (March 2011)

- Lecturer at the JSPS international school on dark energy, Kochi, Japan (August 2010)

- Invited participant at the Benasque workshop on Modern Cosmology, Benasque, Spain (August 2010)

- Lecturer at the Workshop on Observational Cosmology, Tubitak (Turkish) National Observatory, Turkey (October 2009) 